\title{
Exotic projective structures and quasi-Fuchsian space II
}

\author{
Kentaro Ito
}

January 24, 2007

\begin{abstract}
Let $P(S)$ be the space of projective structures on a closed surface $S$ of genus $g>1$ and let $Q(S)$ be the subset of $P(S)$ of projective structures with quasi-Fuchsian holonomy. It is known that $Q(S)$ consists of infinitely many connected components. In this paper, we will show that the closure of any exotic component of $Q(S)$ is not a topological manifold with boundary and that any two components of $Q(S)$ have intersecting closures.
\end{abstract}

\section{Introduction}

Let $S$ be an oriented closed surface of genus $g>1$. A projective structure on $S$ is a $(G, X)$-structure, where $X$ is the Riemann sphere $\mathbb{\mathbb { C }}$ and $G=\mathrm{PSL}_{2}(\mathbb{C})$ is the group of projective automorphisms of $\widehat{\mathbb{C}}$. We consider the space of marked projective structures $P(S)$ on $S$ and its open subset $Q(S)$ of projective structures with quasi-Fuchsian holonomy. It is known that $Q(S)$ consists of infinitely many connected components. The aim of this paper is to study how components of $Q(S)$ lie in $P(S)$, especially how these components bump or self-bump. Here we say that two components $\mathcal{Q}, \mathcal{Q}^{\prime}$ of $Q(S)$ bump if they have intersecting closures and that a component $\mathcal{Q}$ self-bumps if there is a point $Y \in \partial \mathcal{Q}$ such that for any sufficiently small neighborhood $U$ of $Y$ the intersection $U \cap \mathcal{Q}$ is disconnected.

Now let $R(S)$ be the set of conjugacy classes of representations $\rho: \pi_{1}(S) \rightarrow$ $\mathrm{PSL}_{2}(\mathbb{C})$ and $\mathcal{Q F} \subset R(S)$ the subset of faithful representations with quasi-Fuchsian images. It is a result of Hejhal [He] that the holonomy map hol $: P(S) \rightarrow R(S)$, taking a projective structure to its holonomy representation, is a local homeomorphism. Therefore, studying how $Q(S)=h o l^{-1}(\mathcal{Q F})$ lies in $P(S)$ is closely related to studying how the quasi-Fuchsian space $\mathcal{Q F}$ lies in the representation space $R(S)$. It is known by Goldman [Go] that the set of connected components of $Q(S)$ are classified by the set $\mathcal{M} \mathcal{L}_{\mathbb{N}}=\mathcal{M} \mathcal{L}_{\mathbb{N}}(S)$ of integral points of measured laminations; see $\S 2.3-2.4$. We denote by $\mathcal{Q}_{\lambda}$ the component of $Q(S)$ associated to $\lambda \in \mathcal{M} \mathcal{L}_{\mathbb{N}}$, where $\mathcal{Q}_{0}$ is the component of standard projective structures. Here we say that $Y \in Q(S)$ is standard if its developing map is injective; otherwise it is exotic. One of the most important result on the distribution of components of $Q(S)$ in $P(S)$ is obtained by McMullen; see Appendix A in [Mc]: 
Theorem 1.1 (McMullen). There exists a sequence of exotic projective structures which converges to a point in the relative boundary $\partial \mathcal{Q}_{0}=\overline{\mathcal{Q}_{0}}-\mathcal{Q}_{0}$ of the standard component $\mathcal{Q}_{0}$ in $P(S)$.

In fact, given non-zero $\lambda \in \mathcal{M L}_{\mathbb{N}}$, McMullen obtained in [Mc] a bi-infinite convergent sequence

$$
Y_{n} \rightarrow Y_{\infty} \in \partial \mathcal{Q}_{0} \quad(|n| \rightarrow \infty)
$$

associated to $\lambda$ by using the method developed by Anderson and Canary [AC]. In addition, we showed in [It1] that the projective structures $Y_{n}$ above are contained in the exotic component $\mathcal{Q}_{\lambda}$ for all large $|n|$, and thus obtained the following:

Theorem 1.2 (Theorem A in [It1]). For any non-zero $\lambda \in \mathcal{M} \mathcal{L}_{\mathbb{N}}$, we have $\overline{\mathcal{Q}_{0}} \cap \overline{\mathcal{Q}_{\lambda}} \neq$ $\emptyset$.

Now let $\mu$ be a non-zero element of $\mathcal{M} \mathcal{L}_{\mathbb{N}}$ where no curve in its support is isotopic to one in the support of $\lambda$. Then one can obtain the grafting $Z_{\infty}=\operatorname{Gr}_{\mu}\left(Y_{\infty}\right)$ of $Y_{\infty}$ along $\mu$ (see $\S 2.4$ ), which lies in the boundary of $\mathcal{Q}_{\mu}$. Since the map hol is a local homeomorphism, there is a bi-infinite convergent sequence

$$
Z_{n} \rightarrow Z_{\infty} \in \partial \mathcal{Q}_{\mu} \quad(|n| \rightarrow \infty)
$$

which satisfies $h o l\left(Z_{n}\right)=h o l\left(Y_{n}\right)$ for all large $|n|$. Although the sequences $\left\{Y_{n}\right\}_{n \gg 0}$ and $\left\{Y_{n}\right\}_{n \ll 0}$ are contained in the same component $\mathcal{Q}_{\lambda}$, the sequences $\left\{Z_{n}\right\}_{n \gg 0}$ and $\left\{Z_{n}\right\}_{n \ll 0}$ are not necessarily contained in the same component. In fact, the main theorem in this paper (Theorem 1.3 below) states that these sequences are contained in components corresponding to elements $(\lambda, \mu)_{\sharp},(\lambda, \mu)_{b}$ in $\mathcal{M} \mathcal{L}_{\mathbb{N}}$, respectively, which are defined in $\S 2.5$. See Figure 1. We just remark here that $(\lambda, \mu)_{\sharp} \neq(\lambda, \mu)_{b}$ if and only if $i(\lambda, \mu) \neq 0$, where $i(\lambda, \mu)$ is the geometric intersection number of $\lambda$ and $\mu$.

Theorem 1.3. In the same notation as above, we have $\left\{Z_{n}\right\}_{n \gg 0} \subset \mathcal{Q}_{(\lambda, \mu)_{\sharp}}$ and $\left\{Z_{n}\right\}_{n \ll 0} \subset \mathcal{Q}_{(\lambda, \mu)_{b}}$.
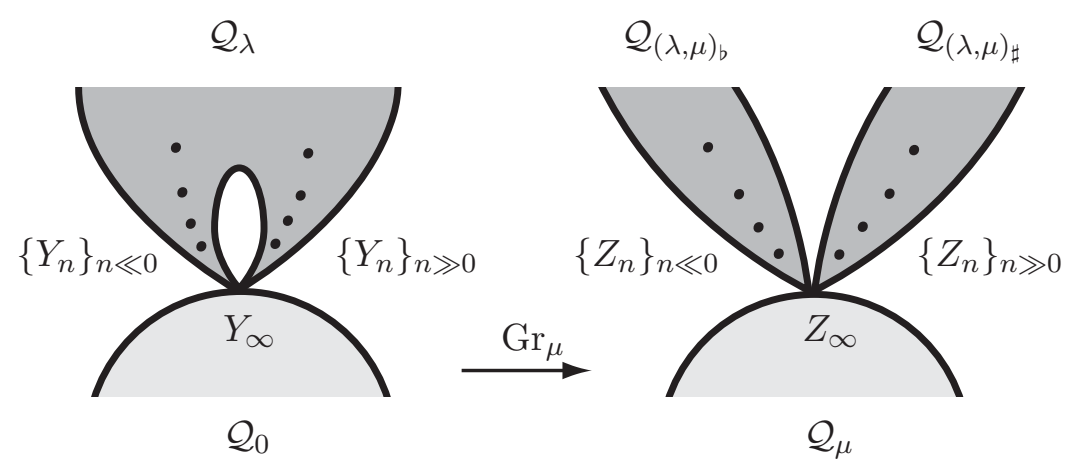

Figure 1: Sequences $\left\{Y_{n}\right\}_{|n| \gg 0}$ and $\left\{Z_{n}\right\}_{|n| \gg 0}$.

As consequences of Theorem 1.3, we obtain the following results on the distribution of components of $Q(S)$; see Theorems 4.1, 4.3 and 4.4. 
Corollary 1.4. (1) For any non-zero $\lambda \in \mathcal{M} \mathcal{L}_{\mathbb{N}}, \mathcal{Q}_{\lambda}$ self-bumps.

(2) For any $\lambda, \mu \in \mathcal{M} \mathcal{L}_{\mathbb{N}}$, we have $\overline{\mathcal{Q}_{\lambda}} \cap \overline{\mathcal{Q}_{\mu}} \neq \emptyset$.

(3) For any non-zero $\lambda \in \mathcal{M} \mathcal{L}_{\mathbb{N}}$, the holonomy map hol $: P(S) \rightarrow R(S)$ is not injective on $\overline{\mathcal{Q}_{\lambda}}$, although it is injective on $\mathcal{Q}_{\lambda}$.

Figure 2 is a computer generated figure by Komori, Sugawa, Wada, and Yamashita (cf. $[\mathrm{KS}]$ and $[\mathrm{KSYW}]$ ), which is a part of a complex one-dimensional slice of $P(S)$ for a punctured torus $S$. All the projective structures in this slice have the same underlying complex structure. The region in white is a slice of $Q(S)$ : the inner disk is a Bers slice (a slice of the standard component $\mathcal{Q}_{0}$ ) and the outer part is a slice of an exotic component. This figure seems to illustrate the phenomenon stated in Theorem 1.3 and Corollary 1.4 (1).

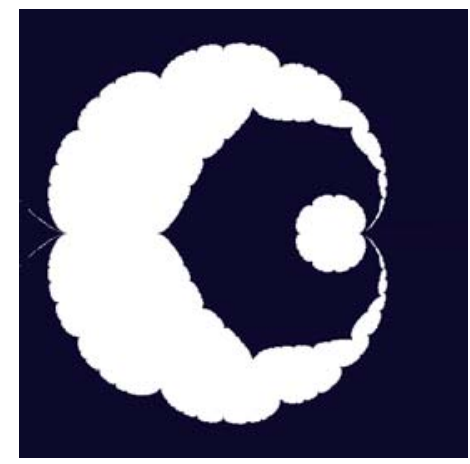

Figure 2: A part of a slice of $Q(S)$ in $P(S)$ (white part) for a punctured torus $S$. An exotic component appears to self-bump and bump the standard component at the right hand side of the inner white disk just like left-hand part of Figure 1.

We now consider associated results on the quasi-Fuchsian space $\mathcal{Q F}$ in $R(S)$. Let $\rho_{n}$ and $\rho_{\infty}$ denote holonomy representations of $Y_{n}$ and $Y_{\infty}$ respectively. Theorem 1.1 then implies that $\mathcal{Q F}$ self-bumps at $\rho_{\infty}$ in $R(S)$; namely, for any sufficiently small neighborhood $U$ of $\rho_{\infty}$ in $R(S)$ the set $U \cap \mathcal{Q F}$ is disconnected; see [Mc]. As a consequence of Theorem 1.3, this result is refined as in the following theorem, which is shown in the proof of Theorem 4.1:

Theorem 1.5. For any sufficiently small neighborhood $U$ of $\rho_{\infty}$ in $R(S), \rho_{n}$ and $\rho_{-n}$ are contained in distinct components of $U \cap \mathcal{Q F}$ for all large enough $n>0$.

Remark. The same result is obtained independently by Bromberg and Holt (oral communication with Bromberg; see also Holt [Ho]).

We mention here the relationship between the topics in this paper and the topology of deformation spaces of Kleinian groups. Let $\Gamma$ be a finitely generated Kleinian group with a non-trivial space $A H(\Gamma)$ of conjugacy classes of discrete faithful representations $\rho: \Gamma \rightarrow \mathrm{PSL}_{2}(\mathbb{C})$. In general, the interior of $A H(\Gamma)$ consists of finitely or 
infinitely many components. It was first shown by Anderson and Canary $[\mathrm{AC}]$ that for some Kleinian group, some of these components bump. This result is generalized by Anderson, Canary and McCullough $[\mathrm{ACM}]$. In our setting, $\Gamma$ is a quasi-Fuchsian group isomorphic to $\pi_{1}(S)$, and the quasi-Fuchsian space $\mathcal{Q F}$ is the interior of the space $A H(\Gamma)$ which consists of exactly one connected component. Also in this case, the idea of Anderson and Canary can be applied to show that $\mathcal{Q F}$ self-bumps by using projective structures; see Theorem 1.1 due to McMullen. In fact, by lifting $\mathcal{Q F} \subset R(S)$ to $Q(S) \subset P(S)$ via the holonomy map hol, we can discuss the bumping and self-bumping of components of $Q(S)$. After McMullen, Bromberg and Holt [BH] characterized the self-bumping of components of the interior of $A H(\Gamma)$ for general Kleinian groups $\Gamma$ without using projective structures. Our results, especially (1) and (2) in Corollary 1.4, can be viewed as the projective structure analogues of the works in $[\mathrm{AC}],[\mathrm{ACM}]$ and $[\mathrm{BH}]$. We refer the reader to $[\mathrm{Ca}]$ for further information on the bumping and self-bumping of deformation spaces of Kleinian groups.

This paper is organized as follows: In section 2, we provide definitions and basic properties of the spaces and maps with which we are concerned. We devote section 3 to the proof of Theorem 1.3. The idea of the proof can be found at the beginning of this section. Corollaries of Theorem 1.3 are obtained in section 4 .

Acknowledgements. The author would like to thank Young-Eun Choi for many useful suggestions to improve the descriptions. He also appreciates the referees for thorough readings of earlier versions of this paper and for many valuable comments and suggestions.

\section{Preliminaries}

We refer the reader to [It1] for more information on some topics in this section. See also an exposition [It2].

\subsection{Kleinian groups}

A Kleinian group $\Gamma$ is a discrete subgroup of $\mathrm{PSL}_{2}(\mathbb{C})$, which acts on the hyperbolic space $\mathbb{H}^{3}$ as isometries, and on the sphere at infinity $S_{\infty}^{2}=\widehat{\mathbb{C}}$ as conformal automorphisms. The region of discontinuity $\Omega(\Gamma)$ is the largest open subset of $\widehat{\mathbb{C}}$ on which $\Gamma$ acts properly discontinuously, and the limit set $\Lambda(\Gamma)$ of $\Gamma$ is its complement $\widehat{\mathbb{C}}-\Omega(\Gamma)$. The quotient manifold $N_{\Gamma}=\left(\mathbb{H}^{3} \cup \Omega(\Gamma)\right) / \Gamma$ is called the Kleinian manifold of $\Gamma$. A quasi-Fuchsian group $\Gamma$ is a Kleinian group whose limit set $\Lambda(\Gamma)$ is a Jordan curve and which contains no element interchanging the two components of $\Omega(\Gamma)$. A b-group $\Gamma$ is a Kleinian group which has exactly one simply connected invariant component of $\Omega(\Gamma)$, which is denoted by $\Omega_{0}(\Gamma)$.

Let $R(S)$ denote the space of all conjugacy classes $[\rho]$ of representations of $\rho$ : $\pi_{1}(S) \rightarrow \mathrm{PSL}_{2}(\mathbb{C})$ such that $\rho\left(\pi_{1}(S)\right)$ is non-abelian. For simplicity, we denote $[\rho]$ by $\rho$ if there is no confusion. The space $R(S)$ is endowed with algebraic topology and is known to be a $(6 g-6)$-dimensional complex manifold (see Theorem 4.21 in [MT] 
for example). quasi-Fuchsian space $\mathcal{Q F}=\mathcal{Q F}(S)$ is the subset of $R(S)$ consisting of faithful representations whose images are quasi-Fuchsian groups. Then it is known by Bers [Be1], Marden [Ma] and Sullivan [Su] that $\mathcal{Q F}$ is connected, contractible and open in $R(S)$.

\section{$2.2 \quad$ Space of projective structures}

A projective structure on $S$ is a $(G, X)$-structure where $X$ is the Riemann sphere $\widehat{\mathbb{C}}$ and $G=\mathrm{PSL}_{2}(\mathbb{C})$ is the group of projective automorphism of $\widehat{\mathbb{C}}$. Let $P(S)$ denote the space of marked projective structures on $S$, or the space of equivalence classes of pairs $(g, Y)$ of a projective surface $Y$ and an orientation preserving homeomorphism $g: S \rightarrow Y$. Two pairs $\left(g_{1}, Y_{1}\right)$ and $\left(g_{2}, Y_{2}\right)$ are said to be equivalent if there is an isomorphism $h: Y_{1} \rightarrow Y_{2}$ of projective structures such that $h \circ g_{1}$ is isotopic to $g_{2}$. The equivalence class of $(g, Y)$ is simply denoted by $Y$.

A projective structure $Y \in P(S)$ has an underlying conformal structure $\pi(Y) \in$ $T(S)$, where $T(S)$ is the Teichmüller space for $S$. The space $P(S)$ is equipped with a structure of a complex $(6 g-6)$-dimensional holomorphic affine bundle over $T(S)$ with the projection $\pi: P(S) \rightarrow T(S)$, see [Ea], [MT, 7.4.1] and [Na, 4.4.6].

A projective structure $Y$ on $S$ can be lifted to a projective structure $\widetilde{Y}$ on $\widetilde{S}$, where $\widetilde{S} \rightarrow S$ is the universal cover on which $\pi_{1}(S)$ acts as a covering group. Since $\widetilde{Y}$ is simply connected, we obtain a developing map $f_{Y}: \widetilde{Y} \rightarrow \widehat{\mathbb{C}}$ by continuing the charts analytically. In addition, the developing map induces a holonomy representation $\rho_{Y}: \pi_{1}(S) \cong \pi_{1}(Y) \rightarrow \mathrm{PSL}_{2}(\mathbb{C})$ which satisfies $f_{Y} \circ \gamma=\rho_{Y}(\gamma) \circ f_{Y}$ for every $\gamma \in \pi_{1}(S)$. We remark that the pair $\left(f_{Y}, \rho_{Y}\right)$ is determined uniquely up to the canonical action of $\mathrm{PSL}_{2}(\mathbb{C})$. We now define the holonomy map

$$
\text { hol }: P(S) \rightarrow R(S)
$$

by $Y \mapsto\left[\rho_{Y}\right]$. Then Hejhal [He] showed that the map hol is a local homeomorphism and Earle $[\mathrm{Ea}]$ and Hubbard $[\mathrm{Hu}]$ independently showed that the map is holomorphic:

Theorem 2.1 (Hejhal, Earle and Hubbard). The holonomy map hol $: P(S) \rightarrow R(S)$ is a holomorphic local homeomorphism.

We denote by $Q(S)=h^{-1}(\mathcal{Q F})$ the set of projective structures with quasiFuchsian holonomy. An element of $Q(S)$ is said to be standard if its developing map is injective; otherwise it is exotic. Let $\mathcal{Q}_{0} \subset Q(S)$ denote the set of all standard projective structures. Given $Y \in \mathcal{Q}_{0}$, the developing map $f_{Y}: \widetilde{Y} \rightarrow \widehat{\mathbb{C}}$ is a homeomorphism onto a connected component of the region of discontinuity $\Omega(\Gamma)$ of the quasi-Fuchsian group $\Gamma=\rho_{Y}\left(\pi_{1}(S)\right)$; thus we have $Y=f_{Y}(\widetilde{Y}) / \Gamma$. It is known by Bers that the map $\left.h o l\right|_{\mathcal{Q}_{0}}: \mathcal{Q}_{0} \rightarrow \mathcal{Q F}$ is a biholomorphism, which takes the Bers' embedded image of the Teichmüller space $T(S)$ in a fiber of $\pi: P(S) \rightarrow T(S)$ to a Bers slice (cf. [Be2]). 


\subsection{Integral points of measured laminations}

Let $\mathcal{S}=\mathcal{S}(S)$ denote the set of homotopy classes of non-trivial simple closed curves on $S$. By abuse of the notation, we also denote a representative of $c \in \mathcal{S}$ by $c$. Let $\mathcal{M} \mathcal{L}_{\mathbb{N}}=\mathcal{M} \mathcal{L}_{\mathbb{N}}(S)$ denote the set of integral points of measured laminations on $S$, or the set of formal summation $\sum_{i=1}^{l} k_{i} c_{i}$ of mutually distinct, disjoint elements $c_{i} \in \mathcal{S}$ with positive integer $k_{i}$ weights. We regard $\mathcal{S} \subset \mathcal{M L}_{\mathbb{N}}$. The "zero-lamination" 0 is also contained in $\mathcal{M} \mathcal{L}_{\mathbb{N}}$. The support of $\lambda=\sum_{i=1}^{l} k_{i} c_{i}$ is denoted by $\underline{\lambda}=\sqcup_{i=1}^{l} c_{i}$. A realization $\hat{\lambda}$ of $\lambda=\sum_{i=1}^{l} k_{i} c_{i} \in \mathcal{M} \mathcal{L}_{\mathbb{N}}$ is a disjoint union of simple closed curves which realize each weighted simple closed curve $k_{i} c_{i}$ by $k_{i}$ parallel disjoint simple closed curves homotopic to $c_{i}$.

For $c, d \in \mathcal{S}$, the geometric intersection number $i(c, d)$ is the minimum number of points in which the representations of $c$ and $d$ must intersect. Note that $i(c, c)=0$ for any $c \in \mathcal{S}$. We naturally extend the definition of the geometric intersection number for elements of $\mathcal{M} \mathcal{L}_{\mathbb{N}}$ by linearity. If $i(\lambda, \mu)=0$ for $\lambda, \mu \in \mathcal{M} \mathcal{L}_{\mathbb{N}}$, we can define $m \lambda+n \mu \in \mathcal{M} \mathcal{L}_{\mathbb{N}}$ for $m, n \in \mathbb{N}$.

\subsection{Grafting}

Let $Y \in P(S)$ and $\widetilde{Y} \rightarrow Y$ the universal cover. A simple closed curve $c \in \mathcal{S}$ is called admissible on $Y$ if a connected component $\widetilde{c} \subset \widetilde{Y}$ of the preimage of $c \subset Y$ is mapped injectively by the developing map $f_{Y}: \widetilde{Y} \rightarrow \widehat{\mathbb{C}}$ onto its image $f_{Y}(\widetilde{c})$ and if the holonomy image $\rho_{Y}(c)$ fixing $f_{Y}(\widetilde{c})$ is loxodromic. We say that $\lambda \in \mathcal{M} \mathcal{L}_{\mathbb{N}}$ is admissible on $Y$ if every component of the support of $\lambda$ is admissible. Note that all $\lambda \in \mathcal{M} \mathcal{L}_{\mathbb{N}}$ are admissible on a standard projective structure $Y \in \mathcal{Q}_{0}$.

Suppose that $c \in \mathcal{S}$ is admissible on $Y \in P(S)$. Let

$$
A_{c}=\left(\widehat{\mathbb{C}}-f_{Y}(\widetilde{c})\right) /\left\langle\rho_{Y}(c)\right\rangle
$$

be the quotient annulus with its induced projective structure. Then the grafting $\operatorname{Gr}_{c}(Y)$ is the projective surface obtained by cutting $Y$ along $c$ and inserting the annulus $A_{c}$ without twisting. More precisely, $A_{c}$ is inserted into $Y-c$ in such a way that the pair of images of a point $x \in \widetilde{c}$ in the boundary of $Y-c$ and the pair of images of $f_{Y}(x) \in f_{Y}(\widetilde{c})$ in the boundary of $A_{c}$ are joined together. Similarly, we can define the grafting $\operatorname{Gr}_{\lambda}(Y)$ for admissible $\lambda \in \mathcal{M} \mathcal{L}_{\mathbb{N}}$ by linearity. The basic fact is that the grafting operation does not change the holonomy representation; i.e. $\operatorname{hol}\left(\operatorname{Gr}_{\lambda}(Y)\right)=\operatorname{hol}(Y)$ holds for every admissible $\lambda \in \mathcal{M} \mathcal{L}_{\mathbb{N}}$.

Goldman [Go] used grafting to classify projective structures with quasi-Fuchsian holonomy:

Theorem 2.2 (Goldman). For every $Y \in Q(S)$ there exist unique $\lambda \in \mathcal{M} \mathcal{L}_{\mathbb{N}}$ and $Y_{0} \in \mathcal{Q}_{0}$ such that $Y=\operatorname{Gr}_{\lambda}\left(Y_{0}\right)$.

Recall that the map $\left.h o l\right|_{\mathcal{Q}_{0}}: \mathcal{Q}_{0} \rightarrow \mathcal{Q F}$ is a biholomorphism. Since the map hol is a local biholomorphism and since hol $\circ \mathrm{Gr}_{\lambda}=$ hol holds on $\mathcal{Q}_{0}$ for a given non-zero $\lambda \in \mathcal{M} \mathcal{L}_{\mathbb{N}}$, the map

$$
\mathrm{Gr}_{\lambda}: \mathcal{Q}_{0} \rightarrow P(S)
$$




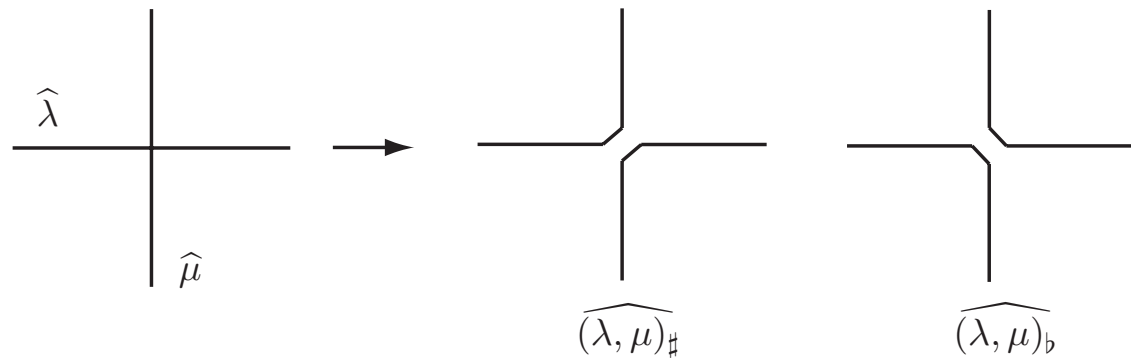

Figure 3: Rules to obtain $(\lambda, \mu)_{\sharp}$ and $(\lambda, \mu)_{b}$.

is a biholomorphism onto its image $\mathcal{Q}_{\lambda}=\operatorname{Gr}_{\lambda}\left(\mathcal{Q}_{0}\right)$. Then, since $\mathcal{Q}_{\lambda}$ is open and connected for every $\lambda \in \mathcal{M} \mathcal{L}_{\mathbb{N}}$, we obtain from Theorem 2.2 the decomposition of $Q(S)$ into its connected components;

$$
Q(S)=\bigsqcup_{\lambda \in \mathcal{M} \mathcal{L}_{\mathbb{N}}} \mathcal{Q}_{\lambda}
$$

\subsection{Operations on $\mathcal{M} \mathcal{L}_{\mathbb{N}}$}

We now introduce two operations

$$
(\cdot, \cdot)_{\sharp},(\cdot, \cdot)_{b}: \mathcal{M} \mathcal{L}_{\mathbb{N}} \times \mathcal{M} \mathcal{L}_{\mathbb{N}} \rightarrow \mathcal{M} \mathcal{L}_{\mathbb{N}},
$$

which is introduced and studied by Luo in [Lu]. For $\lambda, \mu \in \mathcal{M} \mathcal{L}_{\mathbb{N}}$, the elements $(\lambda, \mu)_{\sharp}$ and $(\lambda, \mu)_{b}$ in $\mathcal{M} \mathcal{L}_{\mathbb{N}}$ are obtained as follows: Let $\widehat{\lambda}, \widehat{\mu}$ be realizations of $\lambda, \mu$ whose geometric intersection number is minimal. Next resolve all intersecting points in $\widehat{\lambda} \cup \widehat{\mu}$ as in Figure 3. Then $(\lambda, \mu)_{\sharp}$ and $(\lambda, \mu)_{b}$ are elements in $\mathcal{M L}_{\mathbb{N}}$ whose realizations are the resulting curve systems, respectively; see also Figure 4. We remark that the curves in the integral laminations $\lambda$ and $\mu$ do not need to be oriented for these operations to make sense, but the orientation of the surface is used to distinguish between the two resolutions in Figure 3. We collect in the next lemma some basic properties of these operations, whose proof is left for the reader.

Lemma 2.3. For $\lambda, \mu \in \mathcal{M} \mathcal{L}_{\mathbb{N}}$, we have:

(1) $(\lambda, \mu)_{\sharp}=(\mu, \lambda)_{b}$.

(2) $(\lambda, \mu)_{\sharp}=(\lambda, \mu)_{b}$ if and only if $i(\lambda, \mu)=0$. If $i(\lambda, \mu)=0$ then $(\lambda, \mu)_{\sharp}=$ $(\lambda, \mu)_{b}=\lambda+\mu$.

(3) Suppose that every component of $\mu$ intersects $\lambda$ essentially. Then $\left((\lambda, \mu)_{\sharp}, \mu\right)_{b}=$ $\left((\lambda, \mu)_{b}, \mu\right)_{\sharp}=\lambda$. 


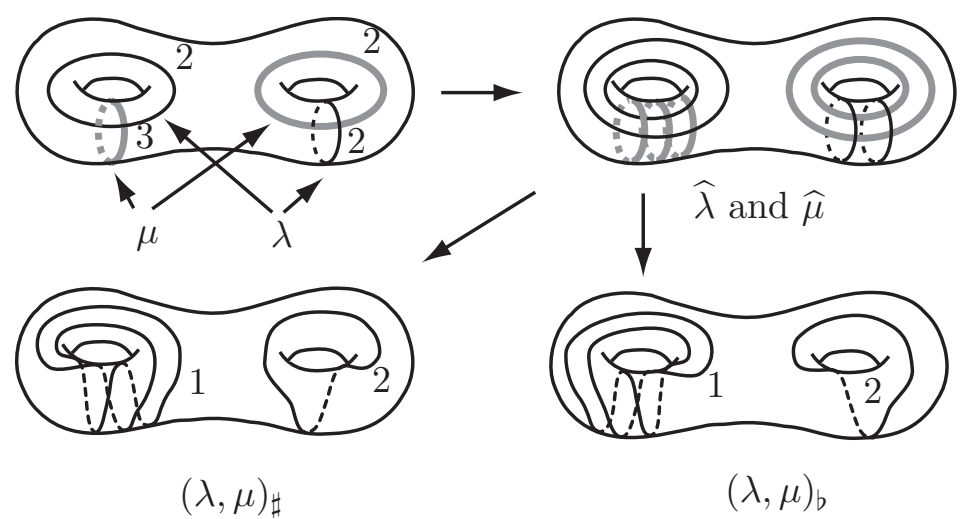

Figure 4: Examples of $(\lambda, \mu)_{\sharp}$ and $(\lambda, \mu)_{b}$.

\subsection{Geometric limits of Kleinian groups}

We begin with the definition of Hausdorff convergence.

Definition 2.4 (Hausdorff convergence, geometric convergence). Let $X$ be a locally compact Hausdorff space. A sequence of closed subsets $A_{n} \subset X$ is said to converge in $X$ to a closed subset $A \subset X$ in the sense of Hausdorff if every element $x \in A$ is the limit of a sequence $\left\{x_{n} \in A_{n}\right\}$ and if every accumulation point of every sequence $\left\{x_{n} \in A_{n}\right\}$ lies in $A$. A sequence of Kleinian groups $\Gamma_{n}$ is said to converge geometrically to a group $\widehat{\Gamma}$ if $\Gamma_{n}$ converges in $\mathrm{PSL}_{2}(\mathbb{C})$ to $\widehat{\Gamma}$ in the sense of Hausdorff.

It is a result of Jørgensen and Marden [JM] that if $\rho_{n} \rightarrow \rho_{\infty}$ in $A H(S)$ then the sequence $\Gamma_{n}=\rho_{n}\left(\pi_{1}(S)\right)$ converges geometrically to a Kleinian group $\widehat{\Gamma}$ up to taking a subsequence. Furthermore, the geometric limit $\widehat{\Gamma}$ always contains the algebraic limit $\Gamma_{\infty}=\rho_{\infty}\left(\pi_{1}(S)\right)$. The following theorem is due to Kerckhoff and Thurston $[\mathrm{KT}]$.

Theorem 2.5 (Kerckhoff-Thurston). Suppose that a sequence $\rho_{n} \in \mathcal{Q F}$ converges to some $\rho_{\infty} \in A H(S)$ and that the sequence $\Gamma_{n}=\rho_{n}\left(\pi_{1}(S)\right)$ converges geometrically to $\widehat{\Gamma}$. Then the sequence $\Lambda\left(\Gamma_{n}\right)$ converges in $\widehat{\mathbb{C}}$ to $\Lambda(\widehat{\Gamma})$ in the sense of Hausdorff.

\subsection{Pullbacks of limit sets of Kleinian groups}

Let $Y \in P(S)$ be a projective structure with discrete faithful holonomy $\rho_{Y}: \pi_{1}(S) \rightarrow$ $\Gamma$. Let $\pi_{Y}: \widetilde{Y} \rightarrow Y$ be the universal cover and let $f_{Y}: \widetilde{Y} \rightarrow \widehat{\mathbb{C}}$ be the developing map. Then the preimage $f_{Y}^{-1}(\Lambda(\Gamma))$ of the limit set $\Lambda(\Gamma)$ in $\widetilde{Y}$ is invariant under the action of the covering transformation group $\pi_{1}(Y)$. Thus the subset $f_{Y}^{-1}(\Lambda(\Gamma))$ in $\widetilde{Y}$ descends to the subset

$$
\Lambda_{Y}:=\pi_{Y} \circ f_{Y}^{-1}(\Lambda(\Gamma))
$$


in $Y$, which is called the pullback of the limit set $\Lambda(\Gamma)$ in $Y$. Similarly, we also obtain the pullback $\pi_{Y} \circ f_{Y}^{-1}(\Lambda(\widehat{\Gamma}))$ of the limit set $\Lambda(\widehat{\Gamma})$ for any Kleinian group $\widehat{\Gamma}$ containing $\Gamma$.

Let $Y=\operatorname{Gr}_{c}\left(Y_{0}\right)$ be the grafting of a standard projective $Y_{0} \in \mathcal{Q}_{0}$ along a simple closed curve $c$. Then the pullback limit set $\Lambda_{Y}$ in $Y$ is a disjoint union of two simple closed curves homotopic to $c$, because the developing image of the universal cover of the grafting annulus hits the limit set twice as it wraps around $\widehat{\mathbb{C}}$ once. By Goldman's grafting theorem (Theorem 2.2), this is generalized as follows:

Lemma 2.6. Let $Y$ be an element of $Q(S)$. Then $Y \in \mathcal{Q}_{\lambda}$ if and only if $\Lambda_{Y} \subset Y$ is a realization of $2 \lambda$.

We now describe the projective analogue of Theorem 2.5. Let $Y_{n} \in Q(S)$ be a sequence converging to some $Y_{\infty} \in \overline{Q(S)}$ as $n \rightarrow \infty$. Then there exist $K_{n}$-bilipschitz maps $\omega_{n}: Y_{\infty} \rightarrow Y_{n}$ with $K_{n} \rightarrow 1$ as $n \rightarrow \infty$ between canonical hyperbolic structures on $Y_{\infty}$ and $Y_{n}$. Let $\rho_{n}$ and $\rho_{\infty}$ denote holonomy representations of $Y_{n}$ and $Y_{\infty}$ respectively and assume that the sequence $\Gamma_{n}=\rho_{n}\left(\pi_{1}(S)\right)$ converges geometrically to a Kleinian group $\widehat{\Gamma}$. Then by Theorem 2.5 the limit sets $\Lambda\left(\Gamma_{n}\right)$ converge to $\Lambda(\widehat{\Gamma})$ in $\widehat{\mathbb{C}}$ in the sense of Hausdorff. In this setting the pullbacks $\Lambda_{Y_{n}} \subset Y_{n}$ of $\Lambda\left(\Gamma_{n}\right)$ converge to the pullback $\widehat{\Lambda}_{Y_{\infty}} \subset Y_{\infty}$ of $\Lambda(\widehat{\Gamma})$ in the sense as follows:

Lemma 2.7 (Lemma 3.3 in [It1]). The sequence $\left\{\omega_{n}^{-1}\left(\Lambda_{Y_{n}}\right)\right\}_{n=1}^{\infty}$ of closed subsets of $Y_{\infty}$ converges to $\widehat{\Lambda}_{Y_{\infty}} \subset Y_{\infty}$ as $n \rightarrow \infty$ in the sense of Hausdorff, where $\widehat{\Lambda}_{Y_{\infty}}=$ $\pi_{Y_{\infty}} \circ f_{Y_{\infty}}^{-1}(\Lambda(\widehat{\Gamma}))$ is the pullback of the limit set $\Lambda(\widehat{\Gamma})$ in $Y_{\infty}$. If there is no confusion, we simply say that $\Lambda_{Y_{n}}$ converge in $Y_{\infty}$ to $\widehat{\Lambda}_{Y_{\infty}}$.

\section{Proof of the main theorem}

We devote this section to the proof of Theorem 1.3. Throughout this proof, Figure 5 should be helpful for the reader to understand the arguments. Here we outline the proof. Given non-zero $\lambda \in \mathcal{M} \mathcal{L}_{\mathbb{N}}$, let

$$
Y_{n} \rightarrow Y_{\infty} \in \partial \mathcal{Q}_{0} \quad(|n| \rightarrow \infty)
$$

be a bi-infinite convergent sequence of exotic projective structures which was constructed by McMullen [Mc] and was shown in [It1] to lie in the component $\mathcal{Q}_{\lambda}$ for all large $|n|$. In $\S 3.1-3.2$, we recall from $[\mathrm{Mc}]$ the construction of the sequence $Y_{n}$ and basic facts of their holonomy representations $\rho_{n}=\operatorname{hol}\left(Y_{n}\right)$. Assume that the sequence $\Gamma_{n}=\rho_{n}\left(\pi_{1}(S)\right)$ converges geometrically to a Kleinian group $\widehat{\Gamma}$. Now let $\mu$ be a non-zero element of $\mathcal{M} \mathcal{L}_{\mathbb{N}}$ where no curve in $\mu$ is isotopic to one in $\underline{\lambda}$. Then we obtain in $\S 3.4$ the grafting $Z_{\infty}=\operatorname{Gr}_{\mu}\left(Y_{\infty}\right)$ of $Y_{\infty}$ along $\mu$ and a bi-infinite convergent sequence

$$
Z_{n} \rightarrow Z_{\infty} \in \partial \mathcal{Q}_{\mu} \quad(|n| \rightarrow \infty)
$$

which satisfies $\operatorname{hol}\left(Z_{n}\right)=\operatorname{hol}\left(Y_{n}\right)$ for all large $|n|$. Our goal is to show that $Z_{n} \in$ $\mathcal{Q}_{(\lambda, \mu)_{\sharp}}$ for all $n \gg 0$ and $Z_{n} \in \mathcal{Q}_{(\lambda, \mu)_{b}}$ for all $n \ll 0$. To this end, it suffices to show 

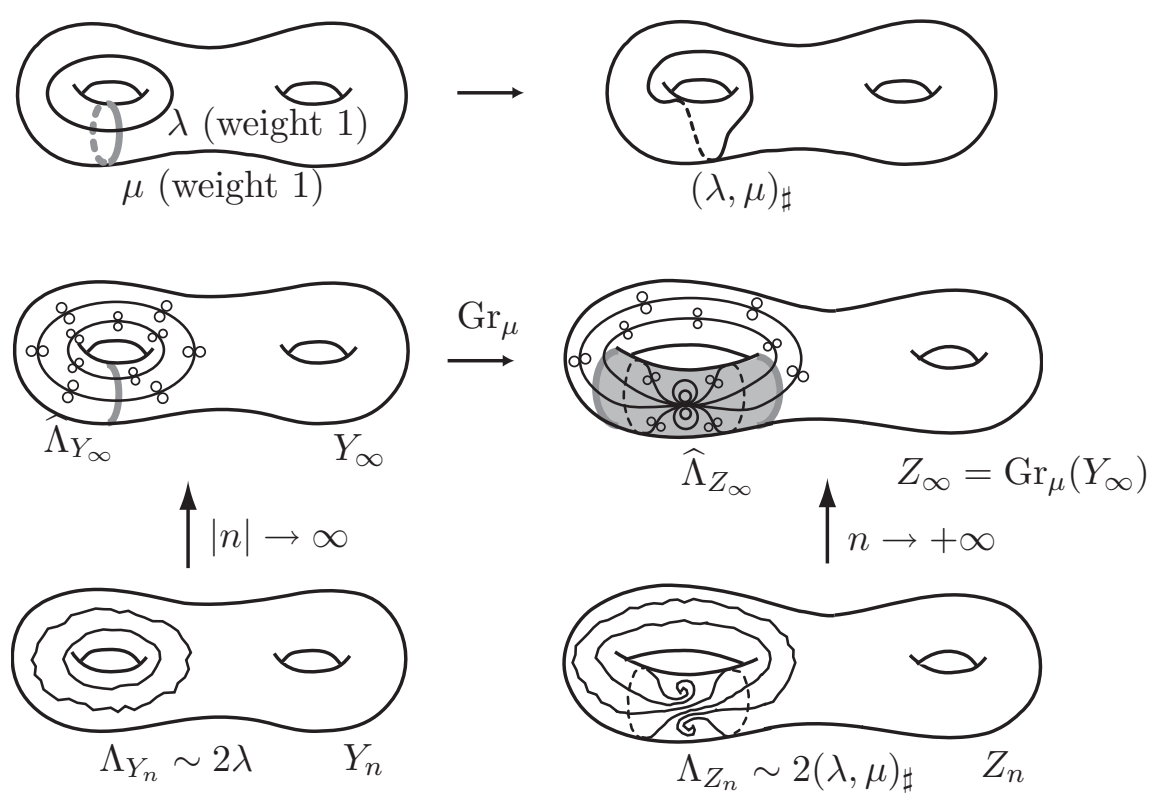

Figure 5: Schematic figure explaining the proof of Theorem 1.3.

from Lemma 2.6 that the pullbacks $\Lambda_{Z_{n}} \subset Z_{n}$ of $\Lambda\left(\Gamma_{n}\right)$ are realizations of $2(\lambda, \mu)_{\sharp}$ for all $n \gg 0$ and of $2(\lambda, \mu)_{b}$ for all $n \ll 0$. Since $\Lambda_{Z_{n}} \subset Z_{n}$ converge to the pullback $\widehat{\Lambda}_{Z_{\infty}} \subset Z_{\infty}$ of $\Lambda(\widehat{\Gamma})$ as $|n| \rightarrow \infty$ in the sense of Lemma 2.7 , in order to understand the shape of $\Lambda_{Z_{n}}$, we first study the shape of $\widehat{\Lambda}_{Z_{\infty}} \subset Z_{\infty}$ in $\S 3.4$ by using the result on the shape of the pullback $\hat{\Lambda}_{Y_{\infty}} \subset Y_{\infty}$ of $\Lambda(\widehat{\Gamma})$ obtained in [It1] and reviewed in $\S 3.3$. In $\S 3.5$ we will see that $\Lambda_{Z_{n}}$ are obtained by resolving $\widehat{\Lambda}_{Z_{\infty}}$ at some points which are pullbacks of rank-two parabolic fixed points in $\Lambda(\widehat{\Gamma})$. The reason for the difference of the resulting curve systems $\Lambda_{Z_{n}}$ for $n \gg 0$ and for $n \ll 0$ is that the limit sets $\Lambda\left(\Gamma_{n}\right)$ for $n \gg 0$ and for $n \ll 0$ are spiraling in opposite directions at each rank-two parabolic fixed point in $\Lambda(\widehat{\Gamma})$, which will be studied closely in $\S 3.5$.

\subsection{A Kleinian group with rank-two parabolic subgroups}

Throughout this section, we fix a non-zero element $\lambda=\sum_{i=1}^{l} k_{i} c_{i} \in \mathcal{M} \mathcal{L}_{\mathbb{N}}$ with its support $\underline{\lambda}=\sqcup_{i=1}^{l} c_{i}$. Let

$$
M_{\lambda}=S \times[-1,1]-\underline{\lambda} \times\{0\}
$$

be a 3-manifold $S \times[-1,1]$ with simple closed curves $c_{i} \times\{0\}(1 \leq i \leq l)$ removed. Let $\widehat{\Gamma}$ be a geometrically finite Kleinian group whose Kleinian manifold $N_{\widehat{\Gamma}}=\left(\mathbb{H}^{3} \cup\right.$ $\Omega(\widehat{\Gamma})) / \widehat{\Gamma}$ is homeomorphic to $M_{\lambda}$. In what follows, we identify $N_{\widehat{\Gamma}}$ with $M_{\lambda}$ via this homeomorphism. Each tubular neighborhood of $c_{i} \times\{0\}$ in $M_{\lambda}$ corresponds to a rank-two cusp end in $N_{\widehat{\Gamma}}$, and hence to a conjugacy class of maximal ranktwo parabolic subgroup $\left\langle\gamma_{i}, \delta_{i}\right\rangle$ in $\widehat{\Gamma} \cong \pi_{1}\left(N_{\widehat{\Gamma}}\right)$. We fix the generators of the group 
$\left\langle\gamma_{i}, \delta_{i}\right\rangle$ so that $\gamma_{i} \in \widehat{\Gamma}$ is freely homotopic to $c_{i} \times\{-1\}$ in $N_{\widehat{\Gamma}}$ and that $\delta_{i} \in \widehat{\Gamma}$ is trivial in $S \times[-1,1]$. Moreover, we orient $\gamma_{i}$ and $\delta_{i}$ so that for some $\theta_{i} \in \mathrm{PSL}_{2}(\mathbb{C})$, $\theta_{i} \circ \gamma_{i} \circ \theta_{i}^{-1}(z)=z+1$ and $\theta_{i} \circ \delta_{i} \circ \theta_{i}^{-1}(z)=z+\tau_{i}$ with $\Im \tau_{i}>0$.

Note that each connected component $\omega$ of $\Omega(\widehat{\Gamma})$ covers a connected component of the conformal boundary $\partial N_{\widehat{\Gamma}}=S \times\{ \pm 1\}$ via the quotient map $\mathbb{H}^{3} \cup \Omega(\widehat{\Gamma}) \rightarrow N_{\widehat{\Gamma}}$, and that the subgroup $\Gamma$ of $\widehat{\Gamma}$ stabilizing $\omega$ is a b-group with $\omega=\Omega_{0}(\Gamma)$.

\subsection{Wrapping maps and associated representations}

We introduce here the wrapping map $w_{\lambda}: S \rightarrow N_{\widehat{\Gamma}}$ associated to $\lambda \in \mathcal{M} \mathcal{L}_{\mathbb{N}}$, which is an immersion determined up to homotopy. For $0 \in \mathcal{M L}_{\mathbb{N}}$, we let $w_{0}: S \rightarrow$ $S \times\{-1 / 2\} \subset N_{\widehat{\Gamma}}$ be the canonical inclusion. The wrapping map $w_{\lambda}: S \rightarrow N_{\widehat{\Gamma}}$ associated to $\lambda \in \sum_{i=1}^{l} k_{i} c_{i} \in \mathcal{M} \mathcal{L}_{\mathbb{N}}$ is an immersion such that the image $w_{\lambda}(S)$ in $N_{\widehat{\Gamma}}$ is obtained by cutting $S \times\{-1 / 2\}$ along $c_{i} \times\{-1 / 2\}$ and inserting an annulus which wraps $k_{i}$ times around $c_{i} \times\{0\}$ in $N_{\widehat{\Gamma}}$ at the cut locus for every $1 \leq i \leq l$; see Figure 6. It is also required that $w_{\lambda}$ is homotopic to $w_{0}$ in $S \times[-1,1]$.

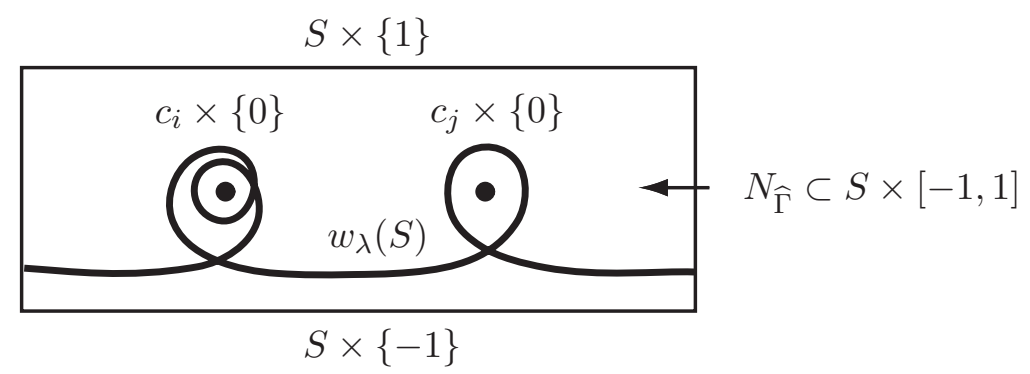

Figure 6: Schematic figure of a wrapping map $w_{\lambda}: S \rightarrow N_{\widehat{\Gamma}}$.

Here we make use of Thurston's Dehn filling theorem ([Co], see also [BO]). By performing simultaneous $(-1, n)$ Dehn filling $(n \in \mathbb{Z})$ on every cusp end of $N_{\widehat{\Gamma}}$, we obtain a sequence of representations $\left\{\chi_{n}: \widehat{\Gamma} \rightarrow \mathrm{PSL}_{2}(\mathbb{C})\right\}$ which satisfies the following:

- $\Gamma_{n}=\chi_{n}(\widehat{\Gamma})$ is a quasi-Fuchsian group,

- The kernel of $\chi_{n}$ is normally generated by $\gamma_{1}^{n} \delta_{1}^{-1}, \ldots, \gamma_{l}^{n} \delta_{l}^{-1}$,

- $\chi_{n}$ converge algebraically to the identity map of $\widehat{\Gamma}$ as $|n| \rightarrow \infty$, and

- $\Gamma_{n}$ converge geometrically to $\widehat{\Gamma}$ as $|n| \rightarrow \infty$.

Then $\chi_{n}\left(\gamma_{i}\right) \rightarrow \gamma_{i}$ and $\chi_{n}\left(\gamma_{i}\right)^{n}=\chi_{n}\left(\gamma_{i}{ }^{n}\right)=\chi_{n}\left(\delta_{i}\right) \rightarrow \delta_{i}$ as $|n| \rightarrow \infty$ for each $1 \leq i \leq l$. Now set

$$
\begin{aligned}
\rho_{n}=\chi_{n} \circ\left(w_{\lambda}\right)_{*}: \pi_{1}(S) \rightarrow \mathrm{PSL}_{2}(\mathbb{C}), \\
\rho_{\infty}=\left(w_{\lambda}\right)_{*}: \pi_{1}(S) \rightarrow \mathrm{PSL}_{2}(\mathbb{C}),
\end{aligned}
$$


where $\left(w_{\lambda}\right)_{*}: \pi_{1}(S) \rightarrow \pi_{1}\left(N_{\widehat{\Gamma}}\right)=\widehat{\Gamma}$ is the group isomorphism induced by $w_{\lambda}$. Then $\rho_{n}$ are faithful representations onto the quasi-Fuchsian groups $\Gamma_{n}$ and the sequence $\rho_{n}$ converges algebraically to $\rho_{\infty}$. One can see that the algebraic limit $\Gamma_{\infty}=\rho_{\infty}\left(\pi_{1}(S)\right)$ is a proper subgroup of the geometric limit $\widehat{\Gamma}$ and is a geometrically finite $b$-group whose Kleinian manifold $N_{\Gamma_{\infty}}$ is homeomorphic to $S \times[-1,1]-\underline{\lambda} \times\{1\}$. Let

$$
Y_{\infty}=\Omega_{0}\left(\Gamma_{\infty}\right) / \Gamma_{\infty}
$$

be the projective structure on the conformal end of $N_{\Gamma_{\infty}}$ corresponding to $S \times\{-1\}$, where $\Omega_{0}\left(\Gamma_{\infty}\right)$ is the invariant component of $\Gamma_{\infty}$. Then $Y_{\infty} \in \partial \mathcal{Q}_{0}$ and $\operatorname{hol}\left(Y_{\infty}\right)=$ $\rho_{\infty}$. Since the map hol is a local homeomorphism, we have a bi-infinite convergent sequence

$$
Y_{n} \rightarrow Y_{\infty} \in \partial \mathcal{Q}_{0} \quad(|n| \rightarrow \infty)
$$

in $Q(S)$ which satisfies $h o l\left(Y_{n}\right)=\rho_{n}$ for all large $|n|$. Then it is known by McMullen (Theorem 1.1) that $Y_{n}$ are exotic for all large $|n|$.

\subsection{The pullback of the limit set in $Y_{\infty}$}

Let $\pi_{Y_{\infty}}: \widetilde{Y}_{\infty}=\Omega_{0}\left(\Gamma_{\infty}\right) \rightarrow Y_{\infty}$ be the universal cover. Then the developing map $f_{Y_{\infty}}: \widetilde{Y}_{\infty}=\Omega_{0}\left(\Gamma_{\infty}\right) \rightarrow \widehat{\mathbb{C}}$ is the identity onto its image. We recall from [It1] the shape of the pullback

$$
\widehat{\Lambda}_{Y_{\infty}}=\pi_{Y_{\infty}} \circ f_{Y_{\infty}}^{-1}(\Lambda(\widehat{\Gamma}))=\left(\Lambda(\widehat{\Gamma}) \cap \Omega_{0}\left(\Gamma_{\infty}\right)\right) / \Gamma_{\infty}
$$

of the limit set $\Lambda(\widehat{\Gamma})$ in $Y_{\infty}$. We first fix our terminology: Let $\xi$ be a finite union of (not necessarily disjoint) simple closed curves on $S$. Then a regular neighborhood $\mathcal{N}(\xi)$ of $\xi$ is a 2-dimensional submanifold of $S$ with boundary and with a deformation retraction $r: \mathcal{N}(\xi) \rightarrow \xi$. Then the following proposition tells us that the pullback limit set $\widehat{\Lambda}_{Y_{\infty}}$ is a "decoration" of a realization of $2 \lambda$ in $Y_{\infty}$ (compare Lemma 2.6).

Proposition 3.1 (Lemma 4.1 in [It1]). There is a regular neighborhood $\mathcal{N}(\widehat{\lambda})$ of a realization $\hat{\lambda}$ of $\lambda$ in $Y_{\infty}$ which satisfy the following:

(1) $\widehat{\Lambda}_{Y_{\infty}}$ is contained in the interior of $\mathcal{N}(\widehat{\lambda})$, and

(2) each connected component $\mathcal{N}$ of $\mathcal{N}(\widehat{\lambda})$ contains exactly two connected components of $\widehat{\Lambda}_{Y_{\infty}}$, each of which contains a simple closed curve homotopic to the core curve of $\mathcal{N}$.

Since $\Lambda_{Y_{n}}$ converge in $Y_{\infty}$ to $\widehat{\Lambda}_{Y_{\infty}}$ as $|n| \rightarrow \infty$ in the sense of Lemma 2.7, we can deduce from Proposition 3.1 that $\Lambda_{Y_{n}}$ are realization of $2 \lambda$ for all large $|n|$. Then it follows from Lemma 2.6 that $Y_{n} \in \mathcal{Q}_{\lambda}$ for all large $|n|$, and thus the result of Theorem 1.2 follows; see [It1] for more details.

In what follows, we will also make use of a regular neighborhood $\mathcal{N}(\underline{\lambda})$ of the support $\underline{\lambda}$ of $\lambda$ which contains $\mathcal{N}(\widehat{\lambda})$, where a connected component $\mathcal{N}\left(c_{i}\right)$ of $\mathcal{N}(\underline{\lambda})$ 
contains $k_{i}$ parallel components of $\mathcal{N}(\widehat{\lambda})$. Throughout this proof of Theorem 1.3, we fix these regular neighborhoods:

$$
\widehat{\Lambda}_{Y_{\infty}} \subset \mathcal{N}(\widehat{\lambda}) \subset \mathcal{N}(\underline{\lambda}) \subset Y_{\infty} .
$$

Note that Proposition 3.1 (1) implies that $\Lambda(\widehat{\Gamma}) \cap \Omega_{0}\left(\Gamma_{\infty}\right)$ lies in $\pi_{Y_{\infty}}^{-1}(\mathcal{N}(\widehat{\lambda}))$, and hence in $\pi_{Y_{\infty}}^{-1}(\mathcal{N}(\underline{\lambda}))$.

\subsection{The pullback of the limit set in $Z_{\infty}$}

Let $\mu$ be a non-zero element of $\mathcal{M} \mathcal{L}_{\mathbb{N}}$ where no curve in $\underline{\mu}$ is isotopic to one in $\underline{\lambda}$. Then $\mu$ is admissible on $Y_{\infty}$ because the developing map $f_{Y_{\infty}}: \widetilde{Y}_{\infty} \rightarrow \Omega_{0}\left(\Gamma_{\infty}\right)$ is injective and every simple closed curve on $S$ mapped by $\rho_{Y_{\infty}}$ to a parabolic element is isotopic to a curve in $\underline{\lambda}$. Thus we obtain the grafting $Z_{\infty}=\operatorname{Gr}_{\mu}\left(Y_{\infty}\right)$ of $Y_{\infty}$ along $\mu$. Furthermore it was shown in [It3] that the map $\operatorname{Gr}_{\mu}: \mathcal{Q}_{0} \rightarrow \mathcal{Q}_{\mu}$ extends continuously to the boundary point $Y_{\infty} \in \partial \mathcal{Q}_{0}$. Thus we have $Z_{\infty} \in \partial \mathcal{Q}_{\mu}$. Although we do not use this fact in this proof of Theorem 1.3, we need this fact in the proof of Theorem 4.3. Since $\operatorname{hol}\left(Y_{\infty}\right)=\operatorname{hol}\left(Z_{\infty}\right)$ and since hol is a local homeomorphism, we have a bi-infinite convergent sequence

$$
Z_{n} \rightarrow Z_{\infty} \in \partial \mathcal{Q}_{\mu} \quad(|n| \rightarrow \infty)
$$

in $Q(S)$ which satisfies $\operatorname{hol}\left(Z_{n}\right)=\operatorname{hol}\left(Y_{n}\right)$ for all large $|n|$.

Let $\pi_{Z_{\infty}}: \widetilde{Z}_{\infty} \rightarrow Z_{\infty}$ be the universal cover. We now study the shape of the pullback

$$
\widehat{\Lambda}_{Z_{\infty}}=\pi_{Z_{\infty}} \circ f_{Z_{\infty}}^{-1}(\Lambda(\widehat{\Gamma}))
$$

of $\Lambda(\widehat{\Gamma})$ in $Z_{\infty}$ by using the observation of the shape of $\widehat{\Lambda}_{Y_{\infty}} \subset Y_{\infty}$ in $\S 3.3$.

Proposition 3.2. There is a regular neighborhood $\mathcal{N}(\widehat{\lambda} \cup \widehat{\mu})$ of $\widehat{\lambda} \cup \widehat{\mu}$ in $Z_{\infty}$ which contains $\widehat{\Lambda}_{Z_{\infty}}$, where $\widehat{\lambda}, \widehat{\mu}$ are realizations of $\lambda, \mu$ in $Z_{\infty}$ whose geometric intersection number is minimal.

We devote the lest of this subsection to the proof of this proposition. For simplicity, we will show in the case where $\mu$ is a simple closed curve $d \in \mathcal{S}$ of weight one. In what follows, we fix a simple closed curve $d$ in $Y_{\infty}$ in its homotopy class. Recall that $\pi_{Y_{\infty}}: \tilde{Y}_{\infty}=\Omega_{0}\left(\Gamma_{\infty}\right) \rightarrow Y_{\infty}$ is the universal cover. Since $d \subset Y_{\infty}$ is admissible, a connected component $\widetilde{d}$ of $\pi_{Y_{\infty}}^{-1}(d)$ in $\Omega_{0}\left(\Gamma_{\infty}\right)$ is $\langle\eta\rangle$-invariant for some loxodromic element $\eta \in \Gamma_{\infty}$. Let

$$
T=(\widehat{\mathbb{C}}-\operatorname{fix}(\eta)) /\langle\eta\rangle
$$

be the quotient torus with its induced projective structure and let

$$
\pi_{T}: \widehat{\mathbb{C}}-\operatorname{fix}(\eta) \rightarrow T
$$

be the covering map. The simple closed curve $\pi_{T}(\widetilde{d})$ in $T$ is also denoted by $d$. We set the longitude $l$ of $T$ as a unique simple closed curve on $T$ (up to homotopy) which is contractible in $\mathbb{H}^{3} /\langle\eta\rangle$, while $d$ is the meridian of $T$. 
Recall that the grafting $Z_{\infty}=\operatorname{Gr}_{d}\left(Y_{\infty}\right)$ is obtained by cutting $Y_{\infty}$ along $d$ and inserting an annulus $T-d$ :

$$
Z_{\infty}=\left(Y_{\infty}-d\right) \cup(T-d)
$$

Then the universal cover $\widetilde{Z}_{\infty}$ of $Z_{\infty}$ is obtained as a multi-sheeted cover of $\widehat{\mathbb{C}}$ by cutting $\widetilde{Y}_{\infty}=\Omega_{0}\left(\Gamma_{\infty}\right)$ along the arc $\widetilde{d}$ and its images under the action of $\Gamma_{\infty}$, and inserting the Riemann sphere $\widehat{\mathbb{C}}-\widetilde{d}$ with a slit, the universal cover of the annulus $T-d$, and its images under the action of $\Gamma_{\infty}$. Then the developing map $f_{Z_{\infty}}: \widetilde{Z}_{\infty} \rightarrow \widehat{\mathbb{C}}$ is just the projection. Let

$$
\widehat{\Lambda}_{T}=\pi_{T}(\Lambda(\widehat{\Gamma})-\operatorname{fix}(\eta))
$$

denote the pullback of $\Lambda(\widehat{\Gamma})$ in $T$. Then we have

$$
\widehat{\Lambda}_{Z_{\infty}}=\left(\widehat{\Lambda}_{Y_{\infty}} \backslash d\right) \cup\left(\widehat{\Lambda}_{T} \backslash d\right),
$$

where $\widehat{\Lambda}_{Y_{\infty}} \backslash d$ and $\widehat{\Lambda}_{T} \backslash d$ are regarded as subsets of $Y_{\infty}-d \subset Z_{\infty}$ and $T-d \subset Z_{\infty}$ respectively.

We now sow that there exist realizations $\hat{\lambda}, \widehat{d}$ of $\lambda, d$ in $Z_{\infty}$ and a regular neighborhood $\mathcal{N}(\widehat{\lambda} \cup \widehat{d}) \subset Z_{\infty}$ of $\widehat{\lambda} \cup \widehat{d}$ containing $\widehat{\Lambda}_{Z_{\infty}}$. Note that there are two copies of the curve $d$ in $Z_{\infty}$. We distinguish between $\widehat{d}$ and those copies of $d$ in $Z_{\infty}$, although $\widehat{d}$ is also a simple closed curve isotopic to one (and hence both) of those copies of $d$ in $Z_{\infty}$.

We first consider the case that $i(\lambda, d)=0$ in $S$. Take $\widehat{\lambda} \subset \mathcal{N}(\widehat{\lambda}) \subset Y_{\infty}$ as in Proposition 3.1. We may assume that $\mathcal{N}(\hat{\lambda})$ is disjoint from $d$ in $Y_{\infty}$. Then $\widehat{\Lambda}_{Y_{\infty}} \cap d=\emptyset$ in $Y_{\infty}$ since $\widehat{\Lambda}_{Y_{\infty}}$ is contained in $\mathcal{N}(\widehat{\lambda})$. Furthermore this also implies that $\Lambda(\widehat{\Gamma}) \cap \widetilde{d}=\emptyset$ in $\widehat{\mathbb{C}}$ and hence that $\widehat{\Lambda}_{T} \cap d=\emptyset$ in $T$. Now let $\mathcal{N}_{T}$ be a closed annulus in $T-d \subset Z_{\infty}$ containing $\widehat{\Lambda}_{T}$ and let $\widehat{d}$ be a core curve of $\mathcal{N}_{T}$. Then we obtain a regular neighborhood

$$
\mathcal{N}(\widehat{\lambda} \sqcup \widehat{d})=\mathcal{N}(\widehat{\lambda}) \sqcup \mathcal{N}_{T}
$$

of $\widehat{\lambda} \sqcup \widehat{d}$ containing $\widehat{\Lambda}_{Z_{\infty}}=\widehat{\Lambda}_{Y_{\infty}} \sqcup \widehat{\Lambda}_{T}$.

We next consider the case that $i(\lambda, d) \neq 0$ in $S$ and set $s=i(\underline{\lambda}, d)$. Here we fix our terminology:

Definition 3.3 (crescent). A closed set $A \subset \widehat{\mathbb{C}}$ is called a crescent if $A$ is the closure of $B_{2}-B_{1}$, where $B_{1}, B_{2}$ are topological closed discs in $\widehat{\mathbb{C}}$ such that $B_{1} \subset B_{2}$ and that $\partial B_{1} \cap \partial B_{2}$ consists of one point $p$. We say that $A$ is touching at $p$. A closed subset in a Riemann surface homeomorphic to a crescent is also called a crescent.

We first consider the shape of $\widehat{\Lambda}_{T}$ in $T$ :

Lemma 3.4. There exist crescents $\left\{\mathcal{A}_{j}\right\}_{j=1}^{s}$ and closed balls $\left\{\mathcal{B}_{j}\right\}_{j=1}^{s}$ in $T$ which satisfy the following (see Figure 7): 
(1) $\mathcal{A}_{1}, \cdots, \mathcal{A}_{s}$ are mutually disjoint. Each $\mathcal{A}_{j}$ is homotopically equivalent to the longitude $l$ in $T$. We let $p_{j} \in T$ denote the touching point of $\mathcal{A}_{j}$.

(2) Interiors of $\mathcal{B}_{1}, \cdots, \mathcal{B}_{s}$ are mutually disjoint.

(3) The set $\bigcup_{j=1}^{s} \mathcal{B}_{j}$ is connected and homotopically equivalent to the meridiand in $T$. In addition, it is satisfied that $\mathcal{A}_{j} \cap\left(\bigcup_{j=1}^{s} \mathcal{B}_{j}\right)=\left\{p_{j}\right\}$ for each $1 \leq j \leq s$, which implies that $\bigcup_{j=1}^{s} \mathcal{B}_{j}$ is a string of beads.

(4) $\widehat{\Lambda}_{T} \subset\left(\bigcup_{j=1}^{s} \mathcal{A}_{j}\right) \cup\left(\bigcup_{j=1}^{s} \mathcal{B}_{j}\right)$.

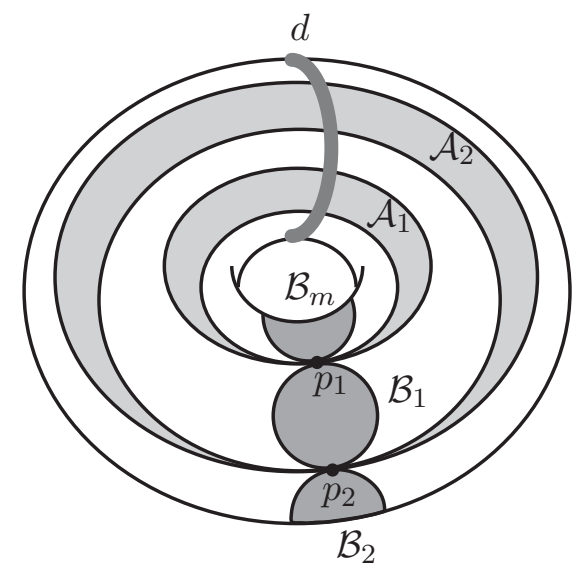

Figure 7: Crescents $\left\{\mathcal{A}_{j}\right\}_{j=1}^{s}$ and balls $\left\{\mathcal{B}_{j}\right\}_{j=1}^{s}$ in $T$.

Proof. We begin with studying the subset $\pi_{T}\left(\Lambda(\widehat{\Gamma}) \cap \Omega_{0}\left(\Gamma_{\infty}\right)\right)$ of $\widehat{\Lambda}_{T}$. Recall from Proposition 3.1 that the set $\Lambda(\widehat{\Gamma}) \cap \Omega_{0}\left(\Gamma_{\infty}\right)$ is contained in the preimage $\pi_{Y_{\infty}}^{-1}(\mathcal{N}(\underline{\lambda}))$ of $\mathcal{N}(\underline{\lambda})$ in $\Omega_{0}\left(\Gamma_{\infty}\right)$. Let $\widetilde{A}$ be a connected component of $\pi_{Y_{\infty}}^{-1}(\mathcal{N}(\underline{\lambda}))$ and let $\gamma \in \Gamma_{\infty}$ be a parabolic element fixing $\widetilde{A}$. Then $\widetilde{A} \cup$ fix $(\gamma)$ is a crescent. Furthermore since the subgroup of $\Gamma_{\infty}$ stabilizing $\widetilde{A}$ is $\langle\gamma\rangle$, it follows that $\eta(\widetilde{A}) \cap \widetilde{A}=\emptyset$ and hence that $\widetilde{A}$ is mapped injectively by $\pi_{T}$ into $T$. If $\widetilde{A} \cap \widetilde{d}=\emptyset$, then the crescent $\widetilde{A} \cup$ fix $(\gamma)$ does not separate two fixed points of $\eta$ and $\pi_{T}(\widetilde{A} \cup$ fix $(\gamma))$ is contractible in $T$. On the other hand, if $\widetilde{A} \cap \widetilde{d} \neq \emptyset$, then $\widetilde{A} \cup$ fix $(\gamma)$ separates two fixed points of $\eta$ and $\pi_{T}(\widetilde{A} \cup$ fix $(\gamma))$ is homotopically equivalent to the longitude $l$ in $T$. Recall that $s=i(\underline{\lambda}, d)$ is the intersection number of $\underline{\lambda}$ and $d$ in $Y_{\infty}$. Then there exist exactly $s$ crescents

$$
\mathcal{A}_{1}, \ldots, \mathcal{A}_{s}
$$

in $T$ each of which is a component of $\pi_{T} \circ \pi_{Y_{\infty}}^{-1}(\mathcal{N}(\underline{\lambda}))$ and is homotopically equivalent to the longitude $l$. Let $p_{j}$ denotes the touching point of $\mathcal{A}_{j}$ for each $j$. Since any 
two components of $\underline{\lambda}$ are not parallel in $Y_{\infty}$, we see that $p_{i} \neq p_{j}$ for $1 \leq i \neq j \leq s$, and hence that $\mathcal{A}_{1}, \cdots, \mathcal{A}_{s}$ are mutually disjoint. We now divide $\widehat{\Lambda}_{T}$ into

$$
\widehat{\Lambda}_{T}=\widehat{\Lambda}_{T}^{\mathcal{A}} \sqcup \widehat{\Lambda}_{T}^{\mathcal{B}},
$$

where $\widehat{\Lambda}_{T}^{\mathcal{A}}=\widehat{\Lambda}_{T} \cap \bigcup_{j=1}^{s} \mathcal{A}_{j}$ and $\widehat{\Lambda}_{T}^{\mathcal{B}}=\widehat{\Lambda}_{T} \backslash \bigcup_{j=1}^{s} \mathcal{A}_{j}$. Observe that the intersection $\widehat{\Lambda}_{T} \cap d$ lies in $\bigcup_{j=1}^{s} \mathcal{A}_{j}$ from the argument above. Then $\widehat{\Lambda}_{T}^{\mathcal{B}}$ is contained in the complement of $d \cup \bigcup_{j=1}^{s} \mathcal{A}_{j}$ in $T$, which is the union of $s$ open balls. Moreover, since $\partial \mathcal{A}_{j}-\left\{p_{j}\right\}$ is disjoint from $\widehat{\Lambda}_{T}$ for each $j$, one see that the intersection of the closure of $\widehat{\Lambda}_{T}^{\mathcal{B}}$ with $\widehat{\Lambda}_{T}^{\mathcal{A}}$ is $\left\{p_{1}, \ldots, p_{s}\right\}$. Therefore, by a slight modification of those open balls, we obtain a string of beads $\bigcup_{j=1}^{s} \mathcal{B}_{j}$ which contains $\widehat{\Lambda}_{T}^{\mathcal{B}}$ and which satisfies the desired conditions.

For each $1 \leq j \leq s=i(\underline{\lambda}, d)$, let $l_{j}$ denote the weight of the component of $\lambda$ associated to $\mathcal{A}_{j}$. Then the crescent $\mathcal{A}_{j}$ contains $l_{j}$ crescents

$$
\mathcal{A}_{j}^{(1)}, \ldots, \mathcal{A}_{j}^{\left(l_{j}\right)}
$$

which are components of $\pi_{T} \circ \pi_{Y_{\infty}}^{-1}(\mathcal{N}(\widehat{\lambda}))$ with common touching point $p_{j}$. Then the statement of Lemma 3.4 is also satisfied with $\mathcal{A}_{j}$ replaced by $\bigcup_{m=1}^{l_{j}} \mathcal{A}_{j}^{(m)}$ for all $j$. Adding suitable (mutually disjoint) closed neighborhoods $V_{j}$ of $p_{j}$ for all $j$, we define a submanifold $\mathcal{N}_{T}$ of $T$ containing $\widehat{\Lambda}_{T}$ by

$$
\mathcal{N}_{T}=\left(\bigcup_{j=1}^{s} V_{j}\right) \cup\left(\bigcup_{j=1}^{s} \bigcup_{m=1}^{l_{j}} \mathcal{A}_{j}^{(m)}\right) \cup\left(\bigcup_{j=1}^{s} \mathcal{B}_{j}\right) .
$$

Then the union

$$
\mathcal{N}(\widehat{\lambda} \cup \widehat{d}):=(\mathcal{N}(\widehat{\lambda}) \backslash d) \cup\left(\mathcal{N}_{T} \backslash d\right)
$$

is a regular neighborhood of $\widehat{\lambda} \cup \widehat{d}$ for suitable realizations $\widehat{\lambda}, \widehat{d}$ of $\lambda, d$ in $Z_{\infty}$. Since $\mathcal{N}(\widehat{\lambda} \cup \widehat{d})$ contains $\widehat{\Lambda}_{Z_{\infty}}=\left(\widehat{\Lambda}_{Y_{\infty}} \backslash d\right) \cup\left(\widehat{\Lambda}_{T} \backslash d\right)$, we obtain the result of Proposition 3.3 in the case of $\mu=d \in \mathcal{S}$. The result for general $\mu \in \mathcal{M} \mathcal{L}_{\mathbb{N}}$ is also obtained by the same argument.

\subsection{Cutting $\mathcal{N}(\widehat{\lambda} \cup \widehat{\mu})$ into $\mathcal{N}\left(\widehat{(\lambda, \mu)_{\sharp}}\right)$ and $\mathcal{N}\left(\widehat{(\lambda, \mu)_{b}}\right)$}

In this subsection, we complete the proof of Theorem 1.3, which states that $Z_{n} \in$ $\mathcal{Q}_{(\lambda, \mu)_{\sharp}}$ for all $n \gg 0$ and $Z_{n} \in \mathcal{Q}_{(\lambda, \mu)_{b}}$ for all $n \ll 0$. In what follows, we mainly consider the case of $n \gg 0$ since the argument for the case $n \ll 0$ is completely parallel. Then, given large enough $n>0$, our goal is to obtain a regular neighborhood $\mathcal{N}\left(\widehat{(\lambda, \mu)_{\sharp}}\right)$ of a realization of $(\lambda, \mu)_{\sharp}$ in $Z_{n}$ containing $\Lambda_{Z_{n}}$ such that each connected component of $\mathcal{N}\left(\widehat{(\lambda, \mu)_{\sharp}}\right)$ contains exactly two connected components of $\Lambda_{Z_{n}}$. Theorem 1.3 then follows from Lemma 2.6. Observe that since there exists a regular neighborhood $\mathcal{N}(\widehat{\lambda} \cup \widehat{\mu})$ of $\widehat{\lambda} \cup \widehat{\mu}$ containing $\widehat{\Lambda}_{Z_{\infty}}$ by Proposition 3.2, and 
since $\Lambda_{Z_{n}}$ converge in $Z_{\infty}$ to $\widehat{\Lambda}_{Z_{\infty}}$ in the sense of Lemma $2.7, \Lambda_{Z_{n}}$ are eventually contained in $\mathcal{N}(\widehat{\lambda} \cup \widehat{\mu})$. Then the desired regular neighborhood $\mathcal{N}\left(\overline{(\lambda, \mu)_{\sharp}}\right)$ is actually obtained by cutting $\mathcal{N}(\widehat{\lambda} \cup \widehat{\mu})$ along a system of simple arcs in $Z_{n}$ which is disjoint from $\Lambda_{Z_{n}}$ (see Figure 8). The existence of such an arc system for large enough $n>0$ is shown in Proposition 3.5 below.
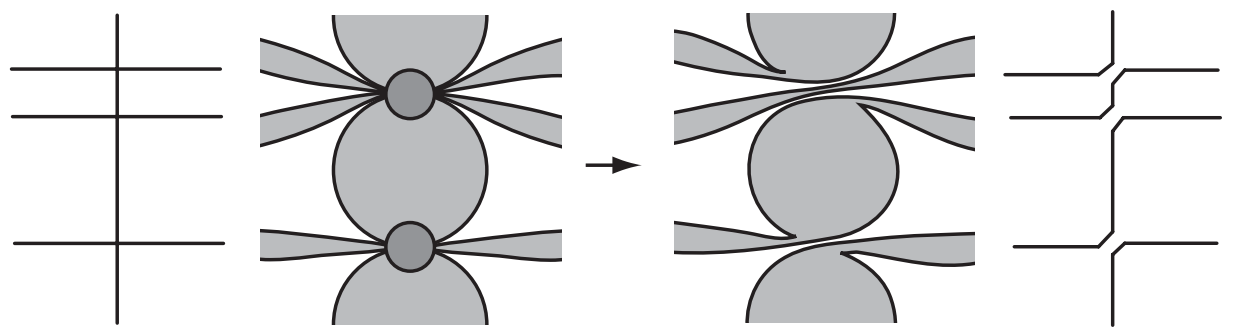

Figure 8: Cutting $\mathcal{N}(\widehat{\lambda} \cup \widehat{\mu})$ into $\mathcal{N}\left(\widehat{(\lambda, \mu)_{\sharp}}\right)$. The graphs at the far left and right illustrate parts of $\widehat{\lambda} \cup \widehat{\mu}$ and $\widehat{(\lambda, \mu)_{\sharp}}$ respectively.

To describe more precisely, we again concentrate our attention to the case of $\mu=d \in \mathcal{S}$ and use the same notation as in $\S 3.4$. Especially $\mathcal{N}(\widehat{\lambda} \cup \widehat{d})$ is a regular neighborhood of $\widehat{\lambda} \cup \widehat{d}$ containing $\widehat{\Lambda}_{Z_{\infty}}$. Note that for each $1 \leq j \leq s$ the point $p_{j} \in \widehat{\Lambda}_{Z_{\infty}}$ is the pullback of a common fixed point $p \in \Lambda(\widehat{\Gamma})$ of a maximal ranktwo parabolic subgroup $\langle\gamma, \delta\rangle$ of $\widehat{\Gamma}$. We suppose that the generators $\gamma, \delta$ satisfy the normalization as in $\S 3.1$. Let $U_{j} \subset Z_{\infty}$ be an open neighborhood of $p_{j}$ containing the closed neighborhood $V_{j}$ of $p_{j}$ and let $\phi_{j}: U_{j} \rightarrow U$ be a homeomorphism from $U_{j} \subset$ $Z_{\infty}$ to an open neighborhood $U \subset \widehat{\mathbb{C}}$ of $p$. We set $V=\phi_{j}\left(V_{j}\right)$. Let $\omega$ be a connected component of $\Omega(\widehat{\Gamma})$ which contains $p$ in its boundary. Then $\omega$ is simply connected and is invariant under the action of $\gamma$. Let $(\omega \cap U)_{+}$and $(\omega \cap U)_{-}$be connected components of $\omega \cap U$ such that $\gamma\left((\omega \cap U)_{+}\right) \subset(\omega \cap U)_{+}$and $\gamma^{-1}\left((\omega \cap U)_{-}\right) \subset(\omega \cap U)_{-}$. Since the components $\delta(\omega)$ and $\delta^{-1}(\omega)$ of $\Omega(\widehat{\Gamma})$ are also $\langle\gamma\rangle$-invariant, we similarly obtain connected components $(\delta(\omega) \cap U)_{+},(\delta(\omega) \cap U)_{-}$of $\delta(\omega) \cap U$ and $\left(\delta^{-1}(\omega) \cap U\right)_{+}$, $\left(\delta^{-1}(\omega) \cap U\right)_{-}$of $\delta^{-1}(\omega) \cap U$. In this situation, we have the following proposition (see Figure 9).

Proposition 3.5. For every large enough $n>0$, there exists a simple arc $\beta_{n}$ in $U$ which satisfies the following:

(1) $\beta_{n} \subset \Omega\left(\Gamma_{n}\right)$,

(2) one end of $\beta_{n}$ lies in $(\omega \cap U)_{+} \backslash V$ and the other end lies in $(\delta(\omega) \cap U)_{-} \backslash V$,

(3) $\beta_{n} \subset(\omega \cap U)_{+} \cup V \cup(\delta(\omega) \cap U)_{-}$, and

(4) $\beta_{n} \cap V$ is connected.

Similarly, for every $n<0$ with large enough $|n|$, there exists a simple arc $\beta_{n}$ in $U$ which satisfies the conditions (1)-(4) above with $\delta(\omega)$ replaced by $\delta^{-1}(\omega)$. 


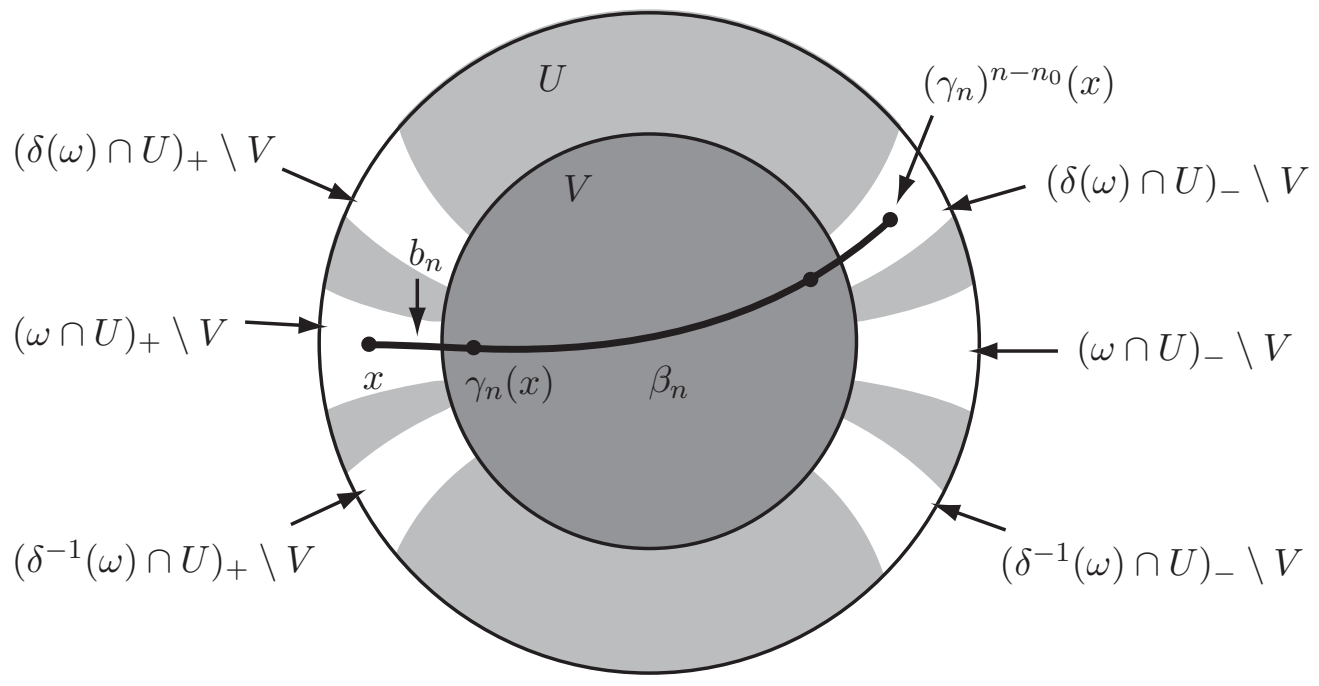

Figure 9: The $\operatorname{arc} \beta_{n}$ constructed in Proposition 3.5.

Before proving the proposition above, We shall complete the proof of Theorem 1.3. We fix $1 \leq j \leq s$ for a while. Recall that $p_{j} \in U_{j}$ is the touching point of $l_{j}$ crescents $A_{j}^{(1)}, \ldots, A_{j}^{\left(l_{j}\right)}$ in $T$. Thus we may assume that $U_{j} \backslash \mathcal{N}(\widehat{\lambda} \cup \widehat{d})$ consists of exactly $2 l_{j}+2$ connected components. Given large enough $n>0$, it follows from Proposition 3.5 that there are mutually disjoint $l_{j}$ simple arcs

$$
\beta_{n}^{(1)}, \ldots, \beta_{n}^{\left(l_{j}\right)}
$$

in $U_{j}$ which satisfy the following:

- $\beta_{n}^{(m)}$ is disjoint from $\Lambda_{Z_{n}}$ for each $1 \leq m \leq l_{j}$,

- the intersection $\beta_{n}^{(m)} \cap \mathcal{N}(\widehat{\lambda} \cup \widehat{d})$ is connected and is contained in $V_{j}$ for each $1 \leq m \leq l_{j}$, and

- all $2 l_{j}$ end points of $\beta_{n}^{(1)}, \ldots, \beta_{n}^{\left(l_{j}\right)}$ are contained in distinct components of $U_{j} \backslash \mathcal{N}(\widehat{\lambda} \cup \widehat{d})$.

Observe that by cutting $\mathcal{N}(\widehat{\lambda} \cup \widehat{d})$ along those arcs in $U_{j}$ for every $1 \leq j \leq$ $s$, we obtain a regular neighborhood $\mathcal{N}\left(\widehat{(\lambda, d)_{\sharp}}\right)$ of a realization of $(\lambda, d)_{\sharp}$ in $Z_{n}$ which contains $\Lambda_{Z_{n}}$; see Figure 8 . On the other hand, since $Z_{n}$ is a quasi-Fuchsian projective structure, Lemma 2.6 implies that $\Lambda_{Z_{n}}$ consists of mutually disjoint nontrivial simple closed curves. Then we claim that each connected component of $\mathcal{N}\left(\widehat{(\lambda, d)_{\sharp}}\right)$ contains exactly two connected components of $\Lambda_{Z_{n}}$, and hence that $\Lambda_{Z_{n}}$ is a realization of $2(\lambda, d)_{\sharp}$. In fact, in the case of $i(\lambda, d) \neq 0$, the claim follows 
from the facts that each connected component of $\mathcal{N}(\widehat{(\lambda, d) \sharp})$ contains a connected component of $\mathcal{N}(\widehat{\lambda}) \backslash d \subset Y_{\infty} \backslash d \subset Z_{\infty}$, and that each connected component of $\mathcal{N}(\widehat{\lambda})$ in $Y_{\infty}$ contains exactly two connected component of $\widehat{\Lambda}_{Y_{\infty}}$ by Proposition 3.1. The proof for the case of $i(\lambda, d)=0$ is left for the reader. In both cases, see [It1] for more rigorous argument. Thus we obtain $Z_{n} \in \mathcal{Q}_{(\lambda, d)_{\sharp}}$ for all $n \gg 0$ by Theorem 2.6. By the same argument, we obtain $Z_{n} \in \mathcal{Q}_{(\lambda, d)}$ b for all $n \ll 0$. This completes the proof of Theorem 1.3 for the case of $\mu=d \in \mathcal{S}$. The result for general $\mu \in \mathcal{M} \mathcal{L}_{\mathbb{N}}$ is obtained by the same argument. It only remains to show Proposition 3.5 in proving Theorem 1.3.

Proof of Proposition 3.5. Recall that there is a sequence $\gamma_{n} \in \Gamma_{n}$ such that $\gamma_{n} \rightarrow \gamma$ and $\left(\gamma_{n}\right)^{n} \rightarrow \delta$ as $n \rightarrow \infty$. Given large enough $n>0$, we will construct the arc $\beta_{n}$ as a union of many images of a short segment by iterations $\gamma_{n}^{k}$ of $\gamma_{n}$.

Choose $x \in(\omega \cap U)_{+} \backslash V$ so that $\gamma^{k}(x) \in \operatorname{int}(V)$ for all $k \in \mathbb{N}$. Let $n_{0}$ be a positive integer such that $\gamma^{-n_{0}} \delta(x) \in(\delta(\omega) \cap U)_{-} \backslash V$ and that $\gamma^{-n_{0}-k} \delta(x) \in \operatorname{int}(V)$ for all $k \in \mathbb{N}$. Since $\gamma_{n}(x) \rightarrow \gamma(x) \in(\omega \cap U)_{+}$as $n \rightarrow \infty$, and since $\Omega\left(\Gamma_{n}\right)$ converge to $\Omega(\widehat{\Gamma})$ in the sense of Carathéodory (see $[\mathrm{KT}])$, one can choose an $\operatorname{arc} b_{n}$ in $(\omega \cap U)_{+} \cap \Omega\left(\Gamma_{n}\right)$ joining $x$ to $\gamma_{n}(x)$ for every large $n$. We now define the arc $\beta_{n}$ by

$$
\beta_{n}=\bigcup_{k=0}^{n-n_{0}-1}\left(\gamma_{n}\right)^{k}\left(b_{n}\right) .
$$

We will see below that $\beta_{n}$, with a slight modification if necessary, satisfies the desired conditions (1)-(4).

(1) Since $b_{n} \subset \Omega\left(\Gamma_{n}\right)$ and $\gamma_{n} \in \Gamma_{n}$, it follows that $\beta_{n} \subset \Omega\left(\Gamma_{n}\right)$.

(2) The arc $\beta_{n}$ joins a point $x \in(\omega \cap U)_{+} \backslash V$ to a point $\left(\gamma_{n}\right)^{n-n_{0}}(x)$, which lies in $(\delta(\omega) \cap U)_{-} \backslash V$ for every large $n$ because $\left(\gamma_{n}\right)^{n-n_{0}}(x)$ converge to $\gamma^{-n_{0}} \delta(x) \in$ $(\delta(\omega) \cap U)_{-} \backslash V$ as $n \rightarrow \infty$. Thus the condition (2) follows.

(3) In order to check that $\beta_{n} \subset(\omega \cap U)_{+} \cup V \cup(\delta(\omega) \cap U)_{-}$for every large $n$, let us consider a subarc $\beta_{n}^{\prime}=\beta_{n}-\left(b_{n} \cup\left(\gamma_{n}\right)^{n-n_{0}}\left(b_{n}\right)\right)$ of $\beta_{n}$, which is obtained from $\beta_{n}$ by removing the first and the last segments. Then we claim that $\beta_{n}^{\prime}$ lies in $V$ for every large $n$. For simplicity, we only show that the orbits $\left\{\left(\gamma_{n}\right)^{k}(x)\right\}_{k=1}^{n-n_{0}-1}$ of $x$ in $\beta_{n}^{\prime}=\bigcup_{k=1}^{n-n_{0}-2}\left(\gamma_{n}\right)^{k}\left(b_{n}\right)$ are contained in $V$. The claim is obtained by the same argument. Now choose a positive integer $N$ so that $\gamma^{s} \delta^{t}(x) \in \operatorname{int}(V)$ holds whenever $|s| \geq N$. We first show that the first $N$ orbits $\left\{\left(\gamma_{n}\right)^{k}(x)\right\}_{k=1}^{N}$ and the last $N$ orbits $\left\{\left(\gamma_{n}\right)^{n-n_{0}-k}(x)\right\}_{k=1}^{N}$ are contained in $V$ for every large $n$. In fact, this is true because for every $1 \leq k \leq N$, we have $\left(\gamma_{n}\right)^{k}(x) \rightarrow \gamma^{k}(x) \in \operatorname{int}(V)$ and $\left(\gamma_{n}\right)^{n-n_{0}-k}(x) \rightarrow \gamma^{n_{0}-k} \delta(x) \in \operatorname{int}(V)$ as $n \rightarrow \infty$.

We next show that the intermediate orbits $\left\{\left(\gamma_{n}\right)^{k}(x)\right\}_{k=N}^{n-n_{0}-N}$ are contained in $V$. To obtain a contradiction, suppose that there is a sequence $k_{n} \rightarrow \infty(n \rightarrow \infty)$ such that $N \leq k_{n} \leq n-n_{0}-N$ and that $\left(\gamma_{n}\right)^{k_{n}}(x) \notin V$. Then passing to a subsequence if necessary, $\left(\gamma_{n}\right)^{k_{n}}(x)$ converges to some point $\hat{x} \notin \operatorname{int}(V)$. Observe that the sequence $\left(\gamma_{n}\right)^{k_{n}}$ does not diverge in $\mathrm{PSL}_{2}(\mathbb{C})$, because both fixed points of $\gamma_{n}$ converge to $p$ and $\left(\gamma_{n}\right)^{k_{n}}(x)$ converge to $\hat{x} \neq p$. Then, since $\left\langle\gamma_{n}\right\rangle$ converge geometrically to 
$\langle\gamma, \delta\rangle$, the sequence $\left(\gamma_{n}\right)^{k_{n}}$ converges in $\mathrm{PSL}_{2}(\mathbb{C})$ to some $\gamma^{s} \delta^{t} \in\langle\gamma, \delta\rangle, s, t \in \mathbb{Z}$ after passing to a further subsequence. Thus we obtain $\hat{x}=\gamma^{s} \delta^{t}(x)$. Since $\hat{x} \notin \operatorname{int}(V)$, it follows that $|s|<N$. On the other hand, since $\left(\gamma_{n}\right)^{k_{n}} \rightarrow \gamma^{s} \delta^{t}$ as $n \rightarrow \infty$, we have $\left(\gamma_{n}\right)^{k_{n}-t n-s} \rightarrow i d$, and thus $k_{n} \equiv t n+s(n \gg 0)$ from Lemma 3.6 in [JM]. It then follows from $N \leq k_{n} \leq n-n_{0}-N$ that $t=0, s \geq 0$ or $t=1, s \leq 0$, both of which contradict to $|s|<N$. Thus the condition (3) follows.

(4) Since the subarc $\beta_{n}^{\prime}$ of $\beta_{n}$ lies in $V$, we can modify $\beta_{n}$ on the first segment $b_{n}$ and the last segment $\left(\gamma_{n}\right)^{n-n_{0}}\left(b_{n}\right)$ so that $\beta_{n} \cap V$ is connected. Thus the condition (4) follows.

The statement for the case of $n<0$ is similarly obtained. This completes the proof of Proposition 3.5.

\section{Corollaries of the main theorem}

We collect here some consequences of Theorem 1.3; see Corollary 1.4.

Theorem 4.1. For any non-zero $\lambda \in \mathcal{M} \mathcal{L}_{\mathbb{N}}$, there exists $Y_{\infty} \in \overline{\mathcal{Q}_{0}} \cap \overline{\mathcal{Q}_{\lambda}}$ such that for any sufficiently small neighborhood $U$ of $Y_{\infty}$ the set $U \cap \mathcal{Q}_{\lambda}$ is disconnected. In particular, $\overline{\mathcal{Q}_{\lambda}}$ is not a topological manifold with boundary.

Proof. We use the same notation as in the beginning of $\S 3$, and suppose that $i(\lambda, \mu) \neq 0$. Since the grafting $Z_{\infty}=\operatorname{Gr}_{\mu}\left(Y_{\infty}\right)$ exists and since the map hol is a local homeomorphism, there exist neighborhoods $V$ of $Y_{\infty}, V^{\prime}$ of $Z_{\infty}$ and $V^{\prime \prime}$ of $\rho_{\infty}=\operatorname{hol}\left(Y_{\infty}\right)=\operatorname{hol}\left(Z_{\infty}\right)$ such that the maps $\left.h o l\right|_{V}: V \rightarrow V^{\prime \prime}$ and $\left.h o l\right|_{V^{\prime}}: V^{\prime} \rightarrow V^{\prime \prime}$ are homeomorphisms. Let $U \subset V$ be a neighborhood of $Y_{\infty}$. Then by Theorem 1.3, for all large $n>0, Z_{n}$ and $Z_{-n}$ are contained in distinct components of $\left(\left.h o l\right|_{V^{\prime}}\right)^{-1}(h o l(U)) \cap Q(S)$. Thus $\rho_{n}$ and $\rho_{-n}$ are contained in distinct components of $h o l(U) \cap \mathcal{Q F}$, which implies Theorem 1.5. Therefore, $Y_{n}$ and $Y_{-n}$ are contained in distinct components of $U \cap \mathcal{Q}_{\lambda}$ for all large $n>0$. Thus the result follows.

We will show in Theorem 4.3 below that any two components of $Q(S)$ bump from Theorem 1.3 combined with the following result obtained in [It1], which is a generalization of Theorem 1.2.

Theorem 4.2 (Theorem B in [It1]). Let $\left\{\lambda_{i}\right\}_{i=1}^{m}$ be a finite subset of $\mathcal{M} \mathcal{L}_{\mathbb{N}}-\{0\}$ such that $i\left(\lambda_{i}, \lambda_{j}\right)=0$ for any $1 \leq i<j \leq m$. Then we have $\overline{\mathcal{Q}_{0}} \cap \overline{\mathcal{Q}_{\lambda_{1}}} \cap \cdots \cap \overline{\mathcal{Q}_{\lambda_{m}}} \neq \emptyset$.

Theorem 4.3. For any $\lambda, \mu \in \mathcal{M} \mathcal{L}_{\mathbb{N}}$, we have $\overline{\mathcal{Q}_{\lambda}} \cap \overline{\mathcal{Q}_{\mu}} \neq \emptyset$.

Proof. Let $\lambda, \mu \in \mathcal{M L}_{\mathbb{N}}$. If $i(\lambda, \mu)=0$, then the result follows from Theorem 4.2. Thus we assume that $i(\lambda, \mu) \neq 0$ and decompose $\mu$ into $\mu=\mu^{\prime}+\mu^{\prime \prime}$ with $\mu^{\prime}, \mu^{\prime \prime} \in \mathcal{M} \mathcal{L}_{\mathbb{N}}$ so that $\mu^{\prime}$ is the union of all curves in $\mu$ which intersect $\lambda$ essentially and that $\mu^{\prime \prime}$ is the union of all curves in $\mu$ which are disjoint from $\lambda$. The proof is divided into the following two cases: (i) $\mu^{\prime \prime}=0$ and (ii) $\mu^{\prime \prime} \neq 0$. 
(i) Case of $\mu^{\prime \prime}=0$. Then $\mu=\mu^{\prime}$. By the same argument as in $\S 3$, replacing $\lambda$ to $(\lambda, \mu)_{b}$, we obtain a sequence

$$
\left\{Y_{n}\right\}_{|n| \gg 0} \subset \mathcal{Q}_{(\lambda, \mu)_{b}}
$$

which converges to some $Y_{\infty} \in \partial \mathcal{Q}_{0}$ as $|n| \rightarrow \infty$. Since no curve in the support of $(\lambda, \mu)_{b}$ is isotopic to one in the support of $\mu$, we obtain the grafting $\operatorname{Gr}_{\mu}\left(Y_{\infty}\right)$ of $Y_{\infty}$ and a convergent sequence

$$
Z_{n} \rightarrow \operatorname{Gr}_{\mu}\left(Y_{\infty}\right) \quad(|n| \rightarrow \infty)
$$

which satisfies $\operatorname{hol}\left(Z_{n}\right)=\operatorname{hol}\left(Y_{n}\right)$ for all large $|n|$. Since $\operatorname{Gr}_{\mu}\left(Y_{\infty}\right) \in \partial \mathcal{Q}_{\mu}$ as mentioned at the beginning of $\S 3.4$, and since $Z_{n} \in \mathcal{Q}_{\left((\lambda, \mu)_{b}, \mu\right)_{\sharp}}=\mathcal{Q}_{\lambda}$ for all $n \gg 0$ by Theorem 1.3, we obtain $\overline{\mathcal{Q}_{\lambda}} \cap \overline{\mathcal{Q}_{\mu}} \neq \emptyset$.

(ii) Case of $\mu^{\prime \prime} \neq 0$. Since $i\left(\lambda, \mu^{\prime \prime}\right)=0$ and $i\left(\mu^{\prime}, \mu^{\prime \prime}\right)=0$, we have $i\left(\left(\lambda, \mu^{\prime}\right)_{b}, \mu^{\prime \prime}\right)=$ 0 . Theorem 4.2 then implies that $\overline{\mathcal{Q}_{0}} \cap \overline{\mathcal{Q}_{\left(\lambda, \mu^{\prime}\right)_{b}}} \cap \overline{\mathcal{Q}_{\mu^{\prime \prime}}} \neq \emptyset$. More precisely, we obtain as in $\S 3$ two sequences

$$
\left\{Y_{n}\right\}_{|n| \gg 0} \subset \mathcal{Q}_{\left(\lambda, \mu^{\prime}\right)_{b}}, \quad\left\{Y_{n}^{\prime}\right\}_{|n| \gg 0} \subset \mathcal{Q}_{\mu^{\prime \prime}}
$$

both of which converge to some $Y_{\infty} \in \partial \mathcal{Q}_{0}$ as $|n| \rightarrow \infty$; see the proof of Theorem B in [It1] for more details. Note that the holonomy image of $Y_{\infty}$ is a $b$-group whose parabolic locus is the support of $\left(\lambda, \mu^{\prime}\right)_{b}+\mu^{\prime \prime}$. Since no curve in the support of $\left(\lambda, \mu^{\prime}\right)_{b}+\mu^{\prime \prime}$ is isotopic to one in the support of $\mu^{\prime}$, we obtain the grafting $\operatorname{Gr}_{\mu^{\prime}}\left(Y_{\infty}\right)$ and two convergent sequences

$$
Z_{n}, Z_{n}^{\prime} \rightarrow \operatorname{Gr}_{\mu^{\prime}}\left(Y_{\infty}\right) \quad(|n| \rightarrow \infty)
$$

which satisfy $\operatorname{hol}\left(Z_{n}\right)=\operatorname{hol}\left(Y_{n}\right)$ and $\operatorname{hol}\left(Z_{n}^{\prime}\right)=\operatorname{hol}\left(Y_{n}^{\prime}\right)$ for all large $|n|$. It follows from Theorem 1.3 that $Z_{n} \in \mathcal{Q}_{\left(\left(\lambda, \mu^{\prime}\right)_{b}, \mu^{\prime}\right)_{\sharp}}=\mathcal{Q}_{\lambda}$ and $Z_{n}^{\prime} \in \mathcal{Q}_{\mu^{\prime}+\mu^{\prime \prime}}=\mathcal{Q}_{\mu}$ for all $n \gg 0$. Thus we obtain $\overline{\mathcal{Q}_{\lambda}} \cap \overline{\mathcal{Q}_{\mu}} \neq \emptyset$ also in this case.

Theorem 4.4. For any non-zero $\lambda \in \mathcal{M L}_{\mathbb{N}}$, the holonomy map hol $: P(S) \rightarrow R(S)$ is not injective on $\overline{\mathcal{Q}_{\lambda}}$, although it is injective on $\mathcal{Q}_{\lambda}$.

Proof. Let $\nu$ be an element of $\mathcal{M} \mathcal{L}_{\mathbb{N}}$ each of whose component intersects $\lambda$ essentially. Lemma 2.3 then implies that

$$
\begin{aligned}
& \left(\nu,(\lambda, \nu)_{\sharp}\right)_{\sharp}=\left((\lambda, \nu)_{\sharp}, \nu\right)_{b}=\lambda, \\
& \left(\nu,(\lambda, \nu)_{b}\right)_{b}=\left((\lambda, \nu)_{b}, \nu\right)_{\sharp}=\lambda .
\end{aligned}
$$

Now let $\left\{Y_{n}\right\}_{|n| \gg 0} \subset Q_{\nu}$ be a sequence constructed as in $\S 3$ which converges to some $Y_{\infty} \in \partial \mathcal{Q}_{0}$ as $|n| \rightarrow \infty$. Applying Theorem 1.3, we obtain two sequences in $\mathcal{Q}_{\lambda}$ as follows:

- $\left\{Z_{n}\right\}_{n \gg 0}$ in $\mathcal{Q}_{\left(\nu,(\lambda, \nu)_{\sharp}\right)_{\sharp}}=\mathcal{Q}_{\lambda}$ which converges to $\operatorname{Gr}_{(\lambda, \nu)_{\sharp}}\left(Y_{\infty}\right)$ as $n \rightarrow+\infty$.

- $\left\{Z_{n}^{\prime}\right\}_{n \ll 0}$ in $\mathcal{Q}_{\left(\nu,(\lambda, \nu)_{b}\right)_{b}}=\mathcal{Q}_{\lambda}$ which converges to $\operatorname{Gr}_{(\lambda, \nu)_{b}}\left(Y_{\infty}\right)$ as $n \rightarrow-\infty$.

Since $i(\lambda, \nu) \neq 0$, we have $(\lambda, \nu)_{\sharp} \neq(\lambda, \nu)_{b}$. Corollary 4.3 in [It3] then implies that $\operatorname{Gr}_{(\lambda, \nu)_{\sharp}}\left(Y_{\infty}\right) \neq \operatorname{Gr}_{(\lambda, \nu)_{b}}\left(Y_{\infty}\right)$ and the result follows. 


\section{References}

[AC] J. W. Anderson and R. D. Canary, Algebraic limits of Kleinian groups which rearrange the pages of a book, Invent. Math. 126 (1996), 205-214.

[ACM] J. W. Anderson, R. D. Canary and D. McCullough, On the topology of deformation spaces of Kleinian groups, Ann. of Math. 152 (2000), 693741.

[Be1] L. Bers, Simultaneous uniformization, Bull. Amer. Math. Soc. 66 (1960), 94-97.

[Be2] L. Bers, On boundaries of Theichmüller spaces and on Kleinian groups, I, Ann. of math. 91 (1970), 570-600.

[BO] F. Bonahon and J. P. Otal, Variétés hyperboliques à géodésiques arbitrairement courtes, Bull. London Math. Soc. 20 (1988), 255-261.

[BH] K. Bromberg and J. Holt, Self-bumping of deformation spaces of hyperbolic 3-manifolds, J. Diff. Geom. 57 (2001), 47-65.

[Ca] R. D. Canary, Pushing the boundary, in the Tradition of Ahlfors and Bers, III, Contemp. Math. 355, Amer. Math. Soc., 2004, 109-121.

[Co] T. D. Comar, Hyperbolic Dehn surgery and convergence of Kleinian groups, Ph. D. Thesis, University of Michigan, 1996.

[Ea] C. J. Earle, On variation of projective structures, Riemann surfaces and related topics, Ann. Math. Studies 97 (1981), 87-99.

[Ga] D. M. Gallo, Deforming real projective structures, Ann. Acad. Sci. Fenn. 22 (1997), 3-14.

[Go] W. M. Goldman, Projective structures with Fuchsian holonomy, J. Diff. Geom. 25 (1987), 297-326.

[He] D. A. Hejhal, Monodromy groups and linearly polymorphic functions, Acta Math. 135 (1975), 1-55.

[Ho] J. Holt, Bumping and self-bumping of deformation spaces, in the Tradition of Ahlfors and Bers, III, Contemp. Math. 355, Amer. Math. Soc., 2004, 269-284.

[Hu] J. H. Hubbard, The monodromy of projective structures, Riemann surfaces and related topics, Ann. Math. Studies 97 (1981), 257-275.

[It1] K. Ito, Exotic projective structures and quasi-Fuchsian space, Duke Math. J. 105 (2000), 185-209. 
[It2] K. Ito, Grafting and components of quasi-fuchsian projective structures, to appear in Spaces of Kleinian groups (eds. Y. Minsky, M. Sakuma, C. Series), London Math. Soc. Lecture note series 329, Cambridge University Press.

[It3] K. Ito, On continuous extensions of grafting maps, Trans. Amer. Math. Soc., to appear.

[JM] T. Jørgensen and A. Marden, Algebraic and geometric convergence of Kleinian groups, Math. Scand. 66 (1990), 47-72.

[KS] Y. Komori and T. Sugawa, Bers embedding of the Teichmuller space of a once-punctured torus, Conform. Geom. Dyn. 8 (2004), 115-142.

[KSYW] Y. Komori, T. Sugawa, Y. Yamashita, M. Wada, Drawing Bers embeddings of the Teichmuller space of once-punctured tori, to appear in Experimental Mathematics.

[KT] S. P. Kerckhoff and W. P. Thurston, Non-continuity of the action of the modular group at Bers' boundary of Teichmuller space, Invent. Math. 100 (1990), 25-47.

[Lu] F. Luo, Some applications of a multiplicative structure on simple loops in surfaces, in Knots, braids, and mapping class groups - papers dedicated to Joan S. Birman (New York, 1998), AMS/IP Stud. Adv. Math., 24, Amer. Math. Soc., Providence, 2001, 123-129.

[Ma] A. Marden, The geometry of finitely generated kleinian groups, Ann. of Math. 99 (1974), 383-462.

[MT] K. Matsuzaki and M. Taniguchi, Hyperbolic manifolds and Kleinian groups, Oxford University Press, 1998.

[Mc] C. T. McMullen, Complex earthquakes and Teichmuller theory, J. Amer. Math. Soc. 11 (1998), 283-320.

[Na] S. Nag, The complex analytic theory of Theichmüller spaces, New York, Wiley 1988.

[Su] D. P. Sullivan, Quasiconformal homeomorphisms and dynamics II: structural stability implies hyperbolicity of Kleinian groups, Acta Math. 155 (1985), 243-260.

Graduate School of Mathematics,

Nagoya University,

Nagoya 464-8602, Japan

itoken@math.nagoya-u.ac.jp 